\title{
De Novo Design of Functional Coassembling Organic-Inorganic Hydrogels for Hierarchical Mineralization and Neovascularization
}

\author{
Babatunde O. Okesola, Ana Karen Mendoza-Martinez, Gianluca Cidonio, Burak Derkus, \\ Delali K. Boccorh, David Osuna de la Peña, Sherif Elsharkawy, Yuanhao Wu, Jonathan I. Dawson, \\ Alastair W. Wark, Dafna Knani, Dave J. Adams, Richard O. C. Oreffo, and Alvaro Mata*
}

Cite This: ACS Nano 2021, 15, 11202-11217

Read Online

\section{ACCESS | Lل|ll Metrics \& More | 回 Article Recommendations | si Supporting Information}

ABSTRACT: Synthetic nanostructured materials incorporating both organic and inorganic components offer a unique, powerful, and versatile class of materials for widespread applications due to the distinct, yet complementary, nature of the intrinsic properties of the different constituents. We report a supramolecular system based on synthetic nanoclay (Laponite, Lap) and peptide amphiphiles (PAs, PAH3) rationally designed to coassemble into nanostructured hydrogels with high structural integrity and a spectrum of

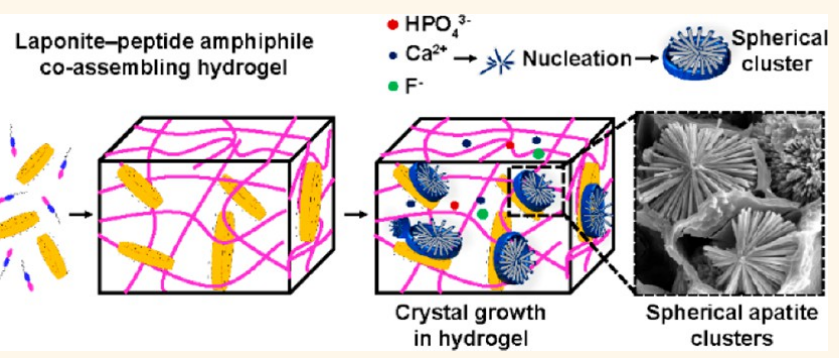
bioactivities. Spectroscopic and scattering techniques and molecular dynamic simulation approaches were harnessed to confirm that PAH3 nanofibers electrostatically adsorbed and conformed to the surface of Lap nanodisks. Electron and atomic force microscopies also confirmed an increase in diameter and surface area of PAH3 nanofibers after coassembly with Lap. Dynamic oscillatory rheology revealed that the coassembled PAH3-Lap hydrogels displayed high stiffness and robust self-healing behavior while gas adsorption analysis confirmed a hierarchical and heterogeneous porosity. Furthermore, this distinctive structure within the three-dimensional (3D) matrix provided spatial confinement for the nucleation and hierarchical organization of high-aspect ratio hydroxyapatite nanorods into well-defined spherical clusters within the 3D matrix. Applicability of the organic-inorganic PAH3-Lap hydrogels was assessed in vitro using human bone marrow-derived stromal cells (hBMSCs) and ex vivo using a chick chorioallantoic membrane (CAM) assay. The results demonstrated that the organic-inorganic PAH3-Lap hydrogels promote human skeletal cell proliferation and, upon mineralization, integrate with the CAM, are infiltrated by blood vessels, stimulate extracellular matrix production, and facilitate extensive mineral deposition relative to the controls.

KEYWORDS: laponite, nanocomposite hydrogels, coassembly, supramolecular, biomineralization, peptide amphiphiles, multicomponent biomaterials

\section{INTRODUCTION}

Nature contains an array of functional nanomaterials that result from the supramolecular coassembly of organic and inorganic building blocks across multiple length scales. Materials such as tooth enamel, bones, nacre from mollusc shells, and marine diatom frustules exhibit a high level of precision over their molecular composition, hierarchical structure, and morphology. The inherent characteristics endow these nanomaterials with properties ranging from high stiffness to light-emission. ${ }^{1,2}$ A fundamental characteristic of natural organic-inorganic composites is the presence of organic matrixes exhibiting ordered arrays of confined charged groups, which induce and regulate the spatial nucleation and hierarchical organization of crystals. $^{3,4}$ These organic components are generally $3 \mathrm{D}$ hydrogel-like materials made from multiple components such as proteins, peptides, polyamines, and polysaccharides. 5,6

This bottom-up "nanofabrication" strategy employed by nature has been harnessed in materials science to design organic-inorganic multicomponent hydrogels with innovative properties. ${ }^{2,7}$ In particular, significant research efforts have been expended to integrate the intrinsic electrical conductivity,

Received: November 23, 2020

Accepted: April 27, 2021

Published: June 28, 2021 
(a)

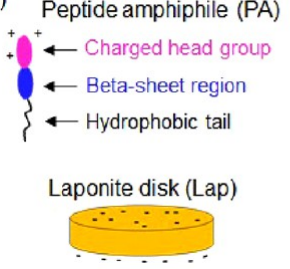

(b)

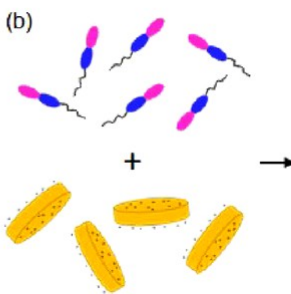

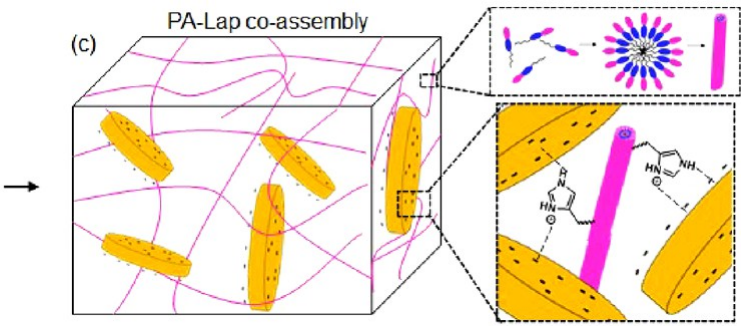

(d)
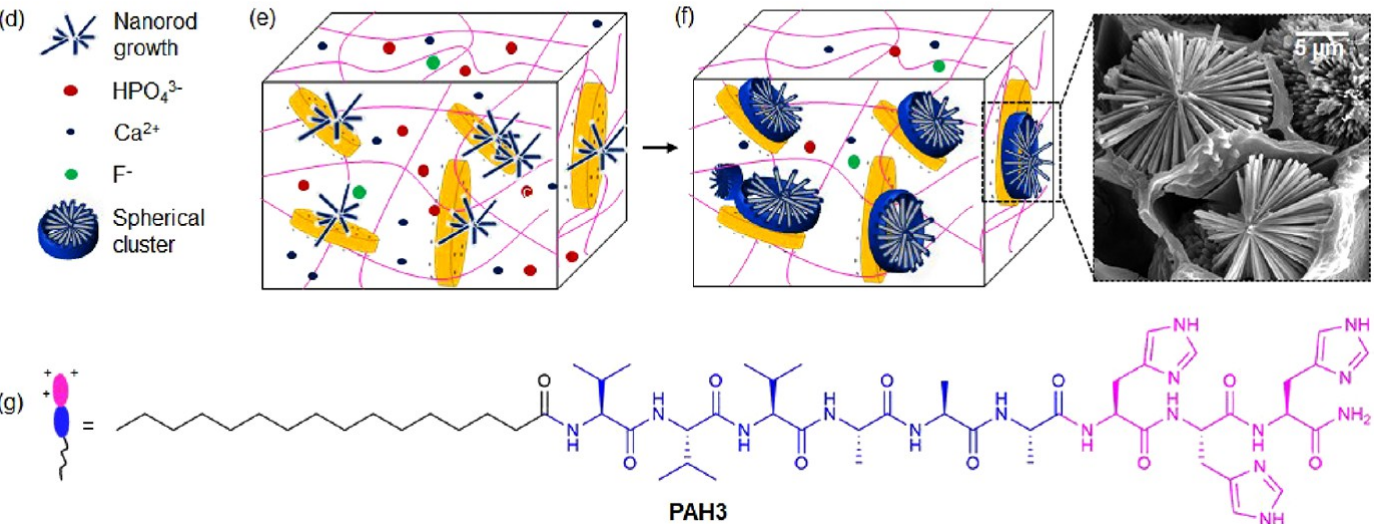

Figure 1. Supramolecular coassembly of exfoliated Lap nanodisks ( - ve) and PA (+ve) to create 3D hydrogels able to guide nucleation and hierarchical growth of hydroxyapatite crystals. (a) Structural representation of a PA with its domains and a Lap nanodisk. (b) Supramolecular coassembly of PAs and Lap to create (c) mechanically robust organic-inorganic hybrid hydrogels with interconnected nanofibers physically cross-linked by Lap nanodisks. Diffusion of (d) mineralizing ionic species into the 3D organic-inorganic hybrid hydrogels triggers the (e) nucleation and (f) hierarchical crystal growth of hydroxyapaptite crystals into high-aspect ratio nanorods organized in spherical clusters. (g) Structural formula for histidine-based PAs.

magnetism, adhesiveness, and hardness of inorganic nanomaterials ${ }^{8}$ with the inherent functionality of both natural (e.g., collagen, ${ }^{9}$ elastin, ${ }^{10}$ DNA, ${ }^{11}$ and hyaluronic acid $\left.^{12}\right)$ and synthetic (e.g., dibenzylidene-d-sorbitol, ${ }^{8,13}$ peptides, ${ }^{7}$ and polymers ${ }^{14}$ ) molecules in the design of advanced organicinorganic hydrogels. These organic-inorganic multicomponent hydrogels are attractive platforms for a wide range of applications in optics, microelectronics, energy storage, catalysis, sensing/environmental cleanup, and nanomedicine. ${ }^{15}$ However, the resulting structures and functions exhibited by these composite materials remain far from those of the natural organic-inorganic materials. ${ }^{16}$

To enhance the properties of organic-inorganic nanocomposites, co-organization of two or more types of inorganic components within the same nanoscale object provides an opportunity to prepare higher-ordered nano-objects with synergistic properties. ${ }^{17}$ Thus, application of such an inorganic approach takes advantage of the distinct properties of the individual inorganics as well as the emergence of new ones that result from their interactions. Current strategies for fabricating organic-inorganic nanocomposites with multi-inorganic nanoobjects are driven by either programmed assembly or reactiondiffusion mechanisms. Programmed assembly involves molecular recognition-driven interparticle aggregation. For example, in a seminal work by Mann and co-workers, DNA-directed attachment of gold nanoparticles to single nanoparticles of silica was used to fabricate discrete nano-objects. ${ }^{18}$ Similarly, barstar-capped iron oxide nanoparticles and barnase-coated quantum dot nanoparticles were coassembled to create superstructures with magnetofluorescence properties. ${ }^{19}$ Other approaches using complementary streptavidin/biotin or antibody/antigen have been harnessed to integrate multiple inorganics in a single organic-inorganic nanocomposite. ${ }^{20}$ In contrast, systems based on reaction-diffusion mechanisms enable assembly of inorganics into nano-objects with spatiotemporal orientation, not readily accessible by equilibrium processes. ${ }^{17,19}$ Examples have been demonstrated in biomineralization, ${ }^{21}$ microfabrication, ${ }^{22-27}$ formation of microlenses, ${ }^{28}$ and dynamic materials. ${ }^{29}$ Reactions of inorganic species, coupled with diffusion in hydrogel media, can lead to the formation of nano-objects with structural hierarchy and complexity as well as multifunctional properties. The rate of formation of these nano-objects within a hydrogel can be controlled by fluid flow, spontaneous compartmentalization, diffusive transport, and Ostwald ripening. ${ }^{30}$

Self-assembling peptides are particularly attractive platforms for the design of organic-inorganic nanostructures, given their intrinsic propensity to assemble into 3D hydrogels, comprising well-defined nanostructures and an ability to display tunable binding affinity for inorganic nanostructures. ${ }^{31}$ These unique attributes of self-assembling peptides have been harnessed to fabricate diverse peptide-inorganic hybrid materials with impressive properties and functionalities. The ability to harness the spatiotemporal organization and enhanced surface chemistry of peptide-inorganic hydrogels would represent a step-change platform to guide crystal morphogenesis in $3 \mathrm{D}$ confinement.?

Laponite XLG (Lap), a trioctahedral synthetic hectorite $\left(\mathrm{Na}^{+}{ }_{0.7}\left[\left(\mathrm{Si}_{8} \mathrm{Mg}_{5.5} \mathrm{Li}_{0.3}\right) \mathrm{O}_{20}(\mathrm{OH})_{4}\right]^{-0.7}\right)$, is a particularly important class of nanosilicate being explored for the design of functional nanomaterials. ${ }^{32}$ Lap displays an ultrathin $2 \mathrm{D}$ nanostructure (diameter $=25-30 \mathrm{~nm}$ and thickness $<1 \mathrm{~nm}$ ), discotic charged surface (permanent negative charge on the surface and positive rim charge), high specific surface area (800 

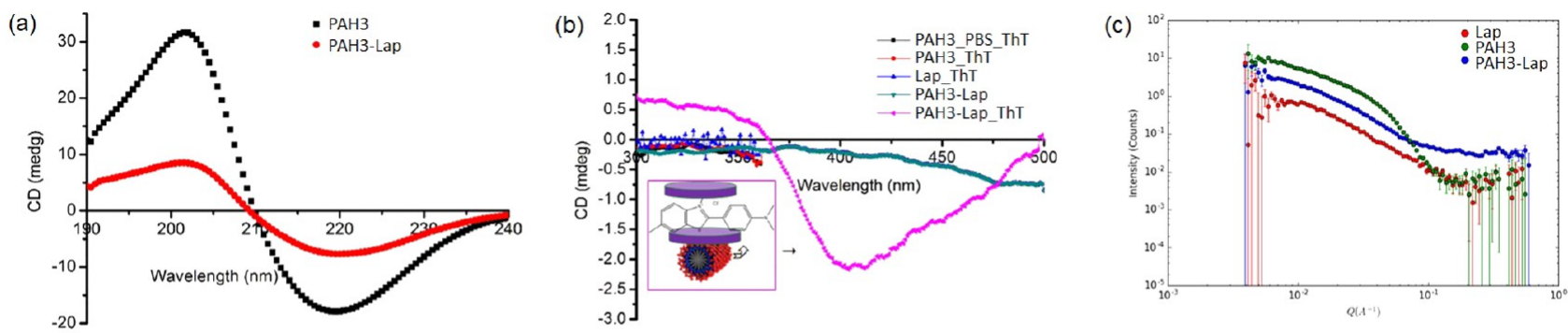

(d)

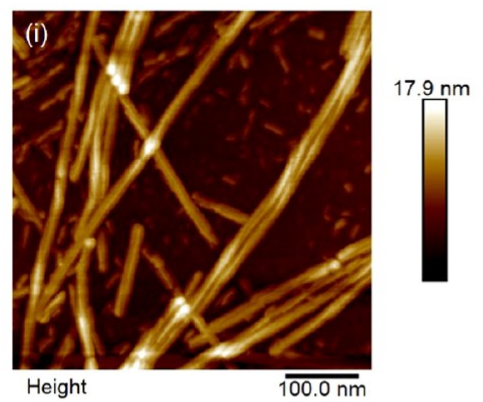

(e)

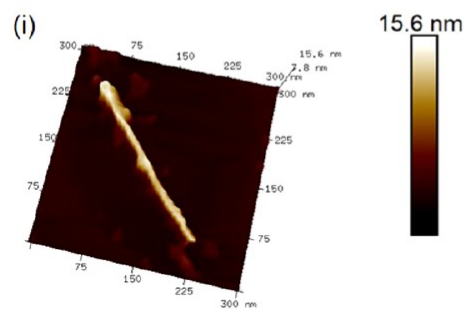

$\mathrm{Ra}=0.81 \mathrm{~nm}, \mathrm{Rq}=1.71 \mathrm{~nm}$

(f) (i)

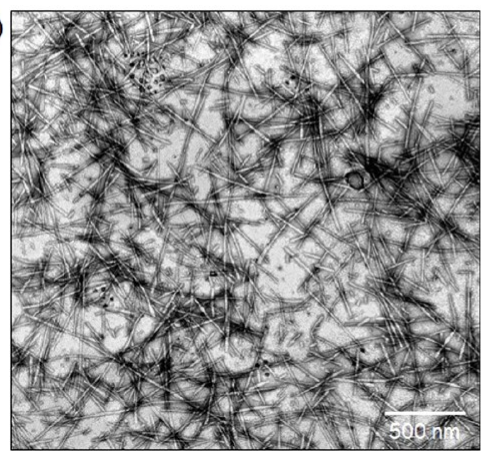

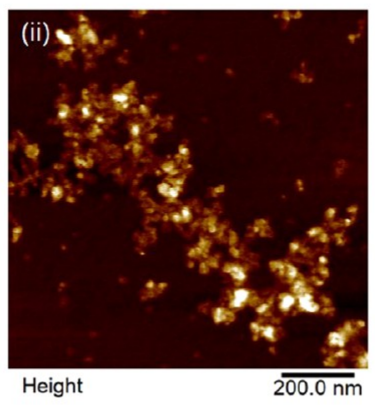

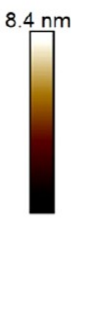

(ii)
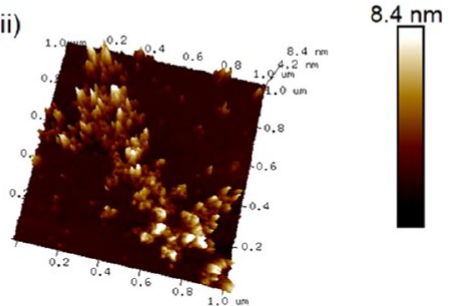

$\mathrm{Ra}=0.83 \mathrm{~nm}, \mathrm{Rq}=1.16 \mathrm{~nm}$

(ii)

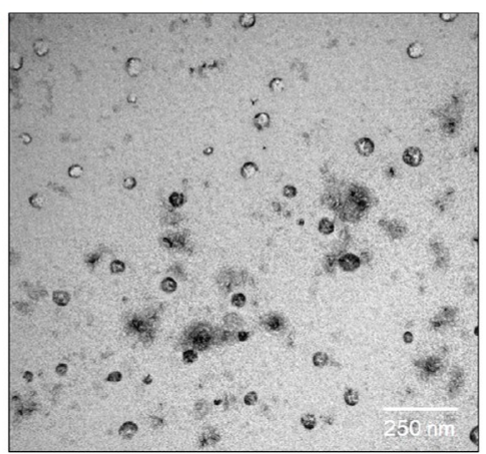

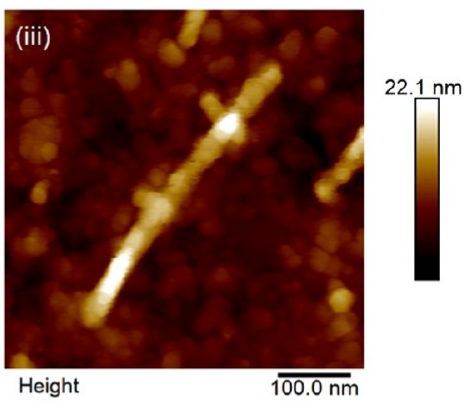
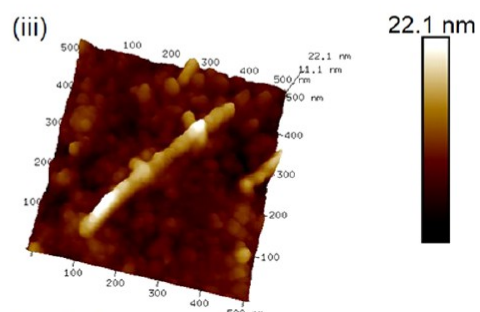

(iii)

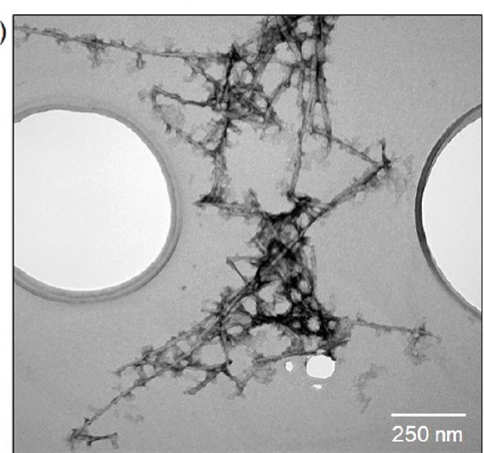

Figure 2. Structural characterization of supramolecular coassembly. (a) CD spectra of an aqueous solution of PAH3 before (square traces) and after (circular traces) adding Lap. (b) Induced CD spectra of thioflavin T (ThT) in the presence of PAH3 in PBS 1x, PAH3-Lap partial hydrogels, and aqueous solution of Lap. The inset represents the proposed mechanism for chirality transfer from PAH3 to Lap nanodisk as a result of supramolecular coassembly, which was confirmed with the use of the molecular rotor ThT. (c) Synchrotron small-angle neutron scattering of PAH3, Lap, and PAH3-Lap coassembly. (d) Atomic force micrographs of (i) PAH3, (ii) Lap, and (iii) PAH3-Lap coassembly as well as the (e) surface topography profile for (i) PAH3, (ii) Lap, and (iii) PAH3-Lap coassembly. (f) Transmission electron micrographs of aqueous suspension of (i) PAH3, (ii) Lap, and (iii) PAH3-Lap.

$\mathrm{m}^{2} / \mathrm{g}$ ), and optical transparency. ${ }^{33}$ Consequently, Lap has been coassembled with synthetic polymers, ${ }^{34-36} \mathrm{DNA}^{37}$ or proteins $^{9,38}$ to develop organic-inorganic hydrogels for numerous biomedical applications and additive manufacturing. ${ }^{32}$ For example, there is a growing interest in the use of polymer-Laponite nanocomposite hydrogels as injectable vehicles for biological cargo including cells, ${ }^{39}$ drug molecules, ${ }^{40}$ and growth factors, ${ }^{41}$ because of their intrinsic shear-thinning property. However, synthetic polymers typically require complex chemical synthesis and purification steps and lack a well-defined structure-property relationship, while natural polymers lack structural tunability and can be difficult to obtain. Therefore, the use of modular and easy-to-synthesize organic building blocks, such as self-assembling peptides, can serve as simpler and more predictable components to interact with and guide the assembly of Lap. Peptide amphiphiles (PAs), a class of self-assembling peptides, have been engineered to facilitate coassembly with biomolecules such as hyaluronic acid, ${ }^{42}$ elastin-like polypeptides, ${ }^{43}$ keratin, ${ }^{44}$ resilinlike polypeptide, ${ }^{45}$ as well as nonpeptidic molecules ${ }^{46}$ to generate different architectures with structural hierarchy and enhanced mechanical and functional properties.

Herein, we report an organic-inorganic nanocomposite hydrogel based on the coassembly of Lap nanodisks with PAs. 
The hydrogels displayed high mechanical strength, shearthinning behavior, and molecular diversity. Furthermore, the resulting PA-Lap coassembled structures served as spatial confinements to guide the formation of nanocrystals with welldefined morphologies across multiple length scales, leading to the formation of multi-inorganic-organic nano-objects (schematically illustrated in Figure 1). These mineralized hydrogels supported cell adhesion, proliferation, differentiation, and neovascularization as assessed by in vitro cell culture and $e x$ vivo using a chick chorioallantoic membrane (CAM) assay.

\section{RESULTS AND DISCUSSION}

Rationale of the Material Design. Our system aims to harness the intrinsic discotic and surface anisotropy of Lap nanodisks and the modularity and self-assembling capacity of PAs to engineer robust and biocompatible hydrogels that not only exhibit the properties of each component but, critically, emergent properties as a result of their coassembly. The PA (PAH3) is a histidine-rich molecule $\left(\mathrm{CH}_{3}-\left(\mathrm{CH}_{2}\right)_{14}-\mathrm{CONH}-\right.$ VVVAAAHHH-CONH , Figure 1g) and is designed to coassemble through interaction with Lap. The unique aromatic imidazole side chain of histidine is key to the self-assembly of proteinaceous fibers driven by organic-inorganic complexation, which is known to generate self-healable and mechanically reinforced biogenic architectures. ${ }^{47}$ We reasoned that the histidine aromatic imidazole side chain $\left(\mathrm{p} K_{\mathrm{a}} \sim 6.0\right)$, which becomes cationic in mildly acidic conditions, would promote electrostatic and intercalation interactions between PAH3 and the negatively charged Lap disk surfaces (surface adsorption). Based on the pioneering work of Aida and coworkers on coassembling Lap nanodisks with guanidiniumbased dendritic binders, ${ }^{48}$ we hypothesized that our PAH3Lap coassembling system would generate mechanically reinforced organic-inorganic hydrogels. It is noteworthy that although cationic PAs with lysine charged head groups have been extensively exploited, self-assembly and gelation of histidine-based PAH3 have yet to be explored. PAK3 $\left(\mathrm{CH}_{3}-\right.$ $\left.\left(\mathrm{CH}_{2}\right)_{14}-\mathrm{CONH}-\mathrm{VVVAAAKKK}-\mathrm{CONH}_{2}\right)$ and PAE3 $\left(\mathrm{CH}_{3}-\right.$ $\left(\mathrm{CH}_{2}\right)_{14}$-CONH-VVVAAAEEE-CONH$\left.{ }_{2}\right)$ were used as controls throughout the experiments.

Mechanism of PAH3-Lap Supramolecular Coassembly. Electrostatic and Sergeant-Soldier Interactions Drive Coassembly. PAs were designed and synthesized as previously reported. ${ }^{49}$ To assess electrostatic interactions between PAH3 and Lap nanodisks, we measured the zeta potential $(\zeta)$ values and hydrodynamic radii $(\mathrm{Rh})$ of individual components against their mixture. The interaction between Lap $(\zeta=-35 \mathrm{mV}$, Rh $=36.42 \pm 2.01 \mathrm{~nm})$ and PAH3 $(\zeta=+25 \mathrm{mV}, \mathrm{Rh}=80.11 \pm$ $4.31 \mathrm{~nm}$ ) evidently revealed the formation of a higher-ordered nanostructure with an increased hydrodynamic radius $(\zeta=$ $-10 \mathrm{mV}, \mathrm{Rh}=115.11 \pm 6.54 \mathrm{~nm}$ ) (Supporting Information Figure $\mathrm{S} 1 \mathrm{a}-\mathrm{e})$. In contrast, the control PAE3 $(-30 \mathrm{mV})$, which exhibits similar net charge as Lap, did not display any interaction, suggesting that the formation of PAH3-Lap was at least partly driven by electrostatic interactions.

Given that PAs are generally known to self-assemble into $\beta$ sheets, we used circular dichroism (CD) spectroscopy to assess interaction between PAH3 and Lap nanodisks. The CD measurements revealed that PAH3 displays a typical $\beta$-sheet conformation with an absorption maximum and minimum at 202 and $218 \mathrm{~nm}$, respectively (Figure 2a). Upon coassembly with Lap, the CD intensities at 202 and $218 \mathrm{~nm}$ decreased by $\sim 16$ and $\sim 11$ mdeg, respectively. This significant decrease in
CD intensities might be due to strong surface adhesion of PAH3 to the Lap nanodisks, resulting in a significant disruption of the PAH3 $\beta$-sheet conformation. Such disruption has been previously reported in silk molecules upon interaction with Lap. ${ }^{37}$ To further confirm surface adsorption of PAH3 to Lap, we used a standard molecular probe thioflavin $\mathrm{T}$ (ThT) which is known to monitor PA self-assembly in aqueous environments. ${ }^{46}$ When the achiral ThT was introduced into a diluted PAH3-Lap hydrogel, a negative band at $410 \mathrm{~nm}$, which is indicative of bound ThT, became apparent (Figure 2b). This absorption band was not observed when ThT was mixed with PAH3 in water, PAH3 in PBS, or Lap in water. Although ThT coassembled with the Lap nanodisk suspension, the Lap-ThT complex exhibited no CD signal, suggesting that ThT acquired an induced chirality due to surface adsorption to Lap nanodisks templated by PAH3 nanofibers (Figure $2 \mathrm{~b}$ inset). Such nanofiber templating of Lap nanodisks has been previously demonstrated with collagen nanofibers due to electrostatic interactions between the positively charged amino acid groups on the periphery of the collagen nanofibrils and negatively charged Lap surfaces. ${ }^{50}$

Nanoscopic Evidence of PA-Lap Coassembly. Nanoscale characterization of the PAH3-Lap composites also confirmed supramolecular integration of both components to generate a higher-ordered nanostructure. First, we used synchrotron small-angle neutron scattering (SANS) to characterize the individual components (Lap and PAH3), as well as their mixture (PAH3-Lap). The SANS data for Lap alone in deuterium oxide $\left(\mathrm{D}_{2} \mathrm{O}\right)$ possesses a $\mathrm{Q}^{-1.8}$ dependency in the range $0.01<0<0.1 \AA^{-1}$, which is consistent with a thin diskshaped structure with a thickness and diameter of $\sim 12$ and $\sim 256 \AA$, respectively (Figure $2 c$, Supporting Information Table S1). The SANS data for PAH3 alone in $\mathrm{D}_{2} \mathrm{O}$ shows the existence of cylinder-like nanostructures with a radius of $\sim 38 \AA$ and several microns in length. The scattering profile of the mixture of Lap and PAH3 shows the coexistence of both disklike nanostructures and cylindrical nanofibers, suggesting a supramolecular coassembly of both nanostructures. The radius of PAH3 nanofibers increased by $\sim 45 \AA$ after coassembly with Lap, suggesting that both components coassembled to form a higher-ordered nanostructure consisting of cylindrical nanofibers and nanodisks. We also confirmed coexistence of Lap disks and PAH3 nanofibers by atomic force microscopy (AFM) (Figure 2d_i-iii). The corresponding surface roughness parameters $\mathrm{Ra}$ and $\mathrm{Rq}$ for the PAH3-Lap nanocomposites $(\mathrm{Ra}=2.12 \mathrm{~nm}, \mathrm{Rq}=3.31 \mathrm{~nm})$ are significantly higher than the values for PAH3 nanofibers $(\mathrm{Ra}=0.81 \mathrm{~nm}, \mathrm{Rq}=1.71 \mathrm{~nm})$ and Lap disks $(\mathrm{Ra}=0.83 \mathrm{~nm}, \mathrm{Rq}=1.16 \mathrm{~nm}$ ) due to the interactions between the two components (Figure 2e i-iii). We used transmission electron microscopy (TEM) and highresolution TEM-energy dispersive spectroscopy (HRTEMEDS) to characterize the coassembly. While TEM (Figure 2f_i-iii) shows the diameters of PAH3 and PAH3-Lap nanofibers to be $\sim 9.8 \mathrm{~nm}$ and $\sim 12 \mathrm{~nm}$, respectively, HRTEMEDS elemental mapping shows colocalization of the characteristic element $(\mathrm{N})$ on PAH3 and the main elemental components $(\mathrm{Si}, \mathrm{Mg}$, and $\mathrm{Na}$ ) of Lap (Supporting Information Figure S2). Such elemental colocalization is consistent with previous studies on Lap and silk coassembly. ${ }^{37}$

Molecular Dynamics Simulations of PA-Lap Coassembly. Structural Optimization of PA-Nanoclay Interactions. In addition to the experimental evidence, we conducted molecular dynamics (MD) simulations (using 
a)
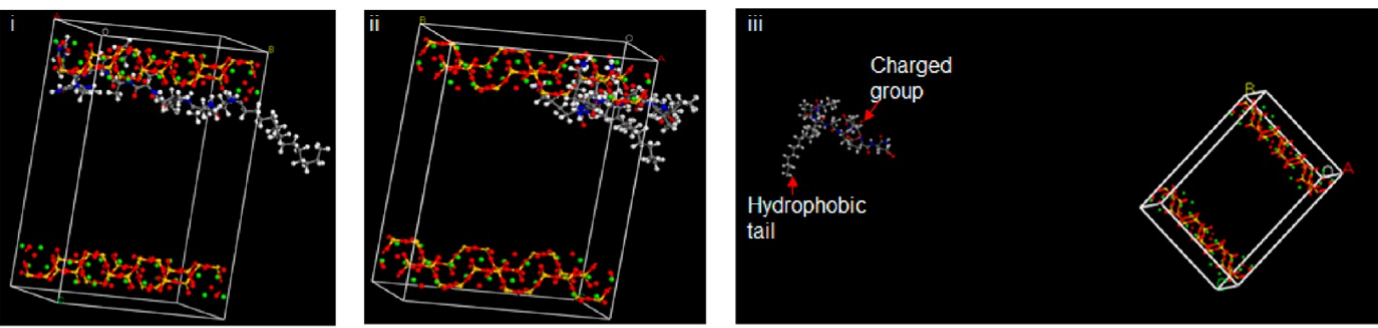

b)
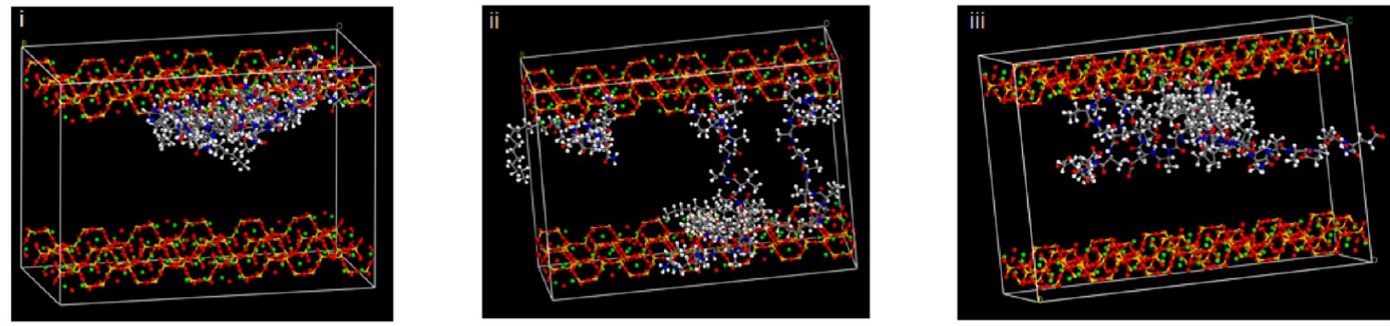

c)
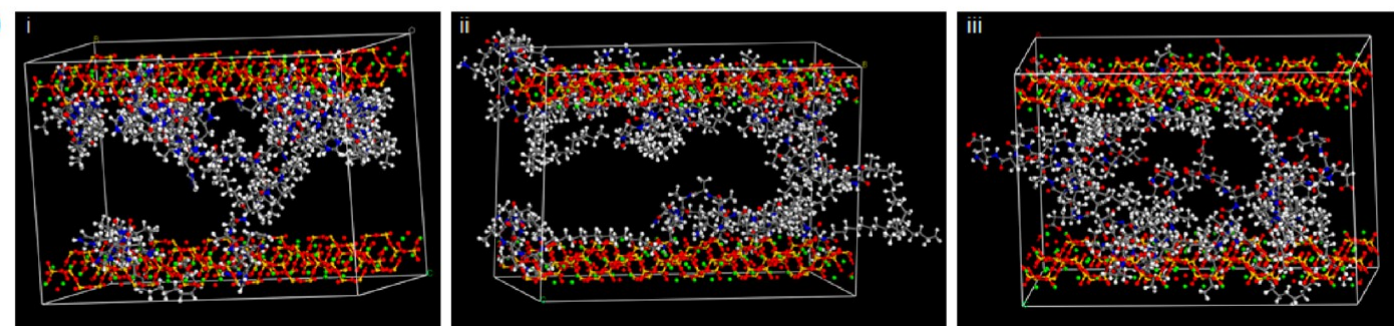

Figure 3. Molecular dynamics simulations of Sepiolite and PAs coassembly. (a) Layered Sepiolite cell with 1 molecule of (i) PAH3, (ii) PAK3, and (iii) PAE3 after 1 ns dynamics steps. (b) Layered Sepiolite Supercell with 4 molecules of (i) PAH3, (ii) PAK3, and (iii) PAE3 after 1 ns dynamics steps. (c) Layered Sepiolite Supercell with 10 molecules of (i) PAH3, (ii) PAK3, and (iii) PAE3 after 1 ns dynamics steps.

Material Studio 8.0 molecular modeling package by Biovia $)^{51}$ to further investigate the mechanism of PA-Lap coassembly. All MD simulations were conducted using the Forcite module with the COMPASS II (condensed-phase optimized molecular potentials for atomistic simulation studies) force field. The molecular structures of PAH3 and controls PAK3 and PAE3 were built and optimized using the visualizer of Materials Studio 8.0. The structure of Lap is not available in the Material Studio 8.0 database; thus, we used Sepiolite $\left(\mathrm{Mg}_{4} \mathrm{Si}_{6} \mathrm{O}_{15}(\mathrm{OH})_{2} \cdot 6 \mathrm{H}_{2} \mathrm{O}\right)$ for this simulation due to its structural similarity with Lap. Like Lap, Sepiolite (Sep) is a layered hydrous magnesium silicate belonging to the $2: 1$ phyllosilicate family and made up of a 2D tetrahedral sheet of $\mathrm{SiO}_{5}{ }^{4-}$. While the slight structural differences between Sep and Lap may be the limit of this simulation, we believe the simulation provides insight into the dynamic interfacial interaction between PAs and layered inorganics. To investigate the interactions between PAs and Sep, first we built two kinds of cells: a small cell with two layers of clay (sepiolite) and one PA molecule and a second cell (Supercell) with enlarged layers with four or ten PA molecules. In this computational elucidation, we considered both electrostatic and van der Waals terms using atom-based summation methods with a repulsive cutoff of $12.5 \AA$. The energies of interaction $\left(E_{\text {inter }}\right)$ of PAH3 with Sep in the cells with one, four, and ten PAH3 molecules are $-736.38,-3060.72$, and $-5626.32 \mathrm{kcal} / \mathrm{mol}$, respectively (Supporting Information Table S2), thus suggesting that the total energy of the PAH3-Sep complex increases as further PAH3 molecules are attracted to Sep to create higherordered nanostructures. In order to isolate the role of the imidazolium side chain of PAH3, we also investigated separately the interactions between a cationic PAK3 and an anionic PAE3 with Sep as controls. While interaction energy values for PAK3-Sep were negative, PAE3-Sep produces positive interaction energy. In both cases, these values increase with increasing number of molecules, suggesting that like PAH3, PAK3 is attracted to the Sep surfaces while PAE3 is strongly repelled.

Force Field MD Simulation Shows Stronger Interaction between Lap and PAH3. Upon insertion of the PA molecules into supercells containing Sep nanoclay, we further confirmed the spatiotemporal orientation of the PA molecules within the lattice $(26.80 \times 53.60 \times 37.64 \AA)$ of the nanoclay. With one molecule in a small cell, PAH3 and PAK3 molecules were attracted to the nanoclay and oriented with their positively charged head groups in close proximity to the layer while the hydrophobic tails are displayed toward the space between the layers (Figure 3a_i-iii). As revealed in the supercells, the PAH3 molecules accumulated on the surface of the nanoclay with the charged imidazolium side chain of the histidine residue facing the nanoclay surface (Figure 3b_i, c_i). In contrast, the PAK3 molecules were more evenly distributed within the lattice (Figure 3b_ii, c_ii). The MD simulation also reveals that the negatively charged headgroups of PAE3 molecules were facing toward the center of the supercells while the hydrophobic tails accumulated on the surface of the nanoclay (Figure 3b_iii, c iii). Taken together, there was no observable interaction between the negatively charged PAE3 and the nanoclay, whereas the cationic PAH3 and PAK3 preferentially interacted with the nanoclay surfaces through the 
(a)
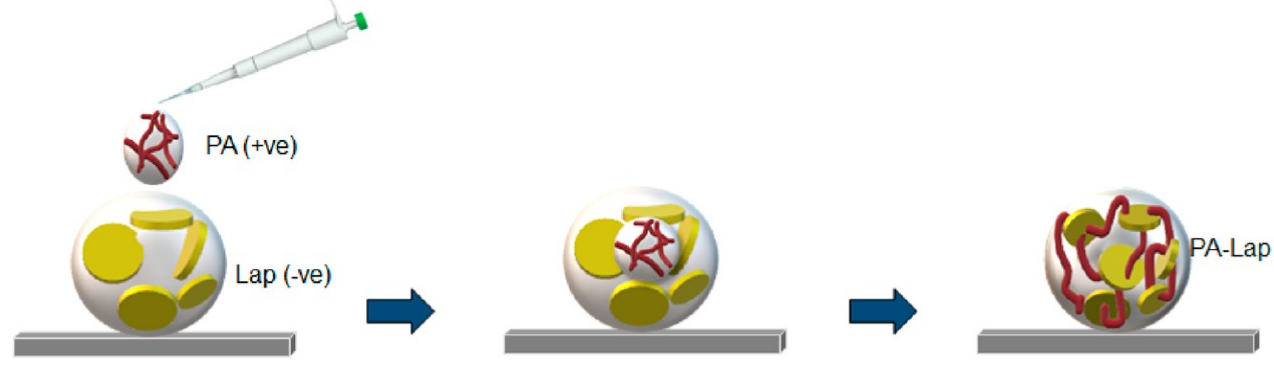

(b)

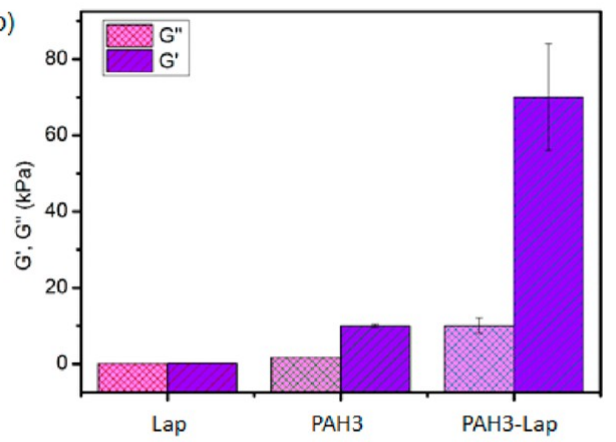

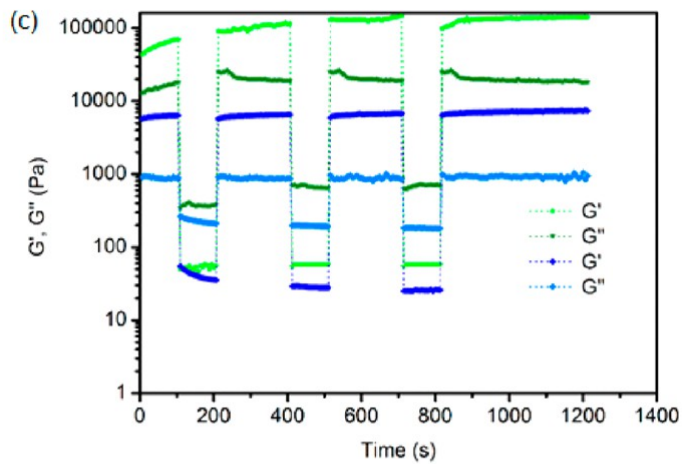

(d)

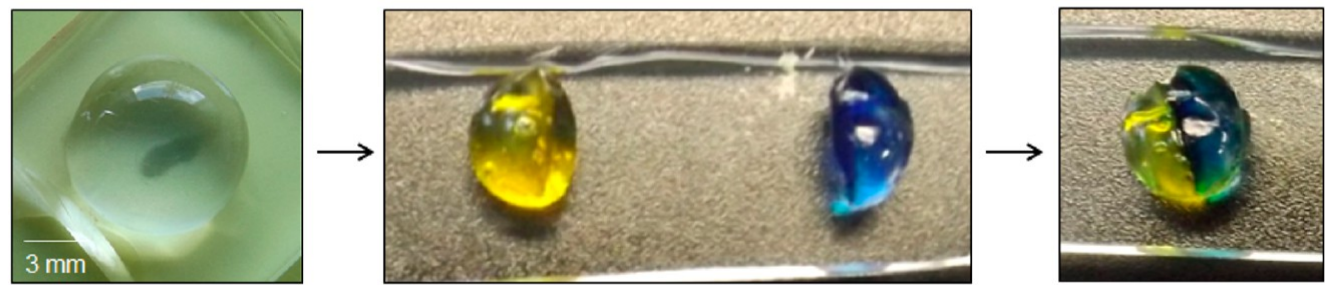

(e)

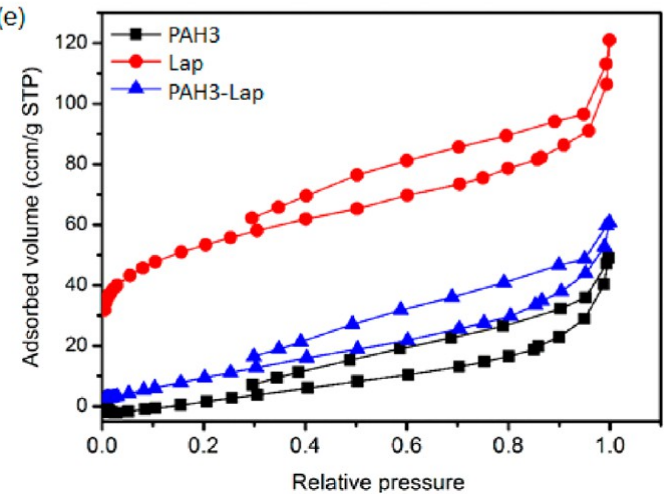

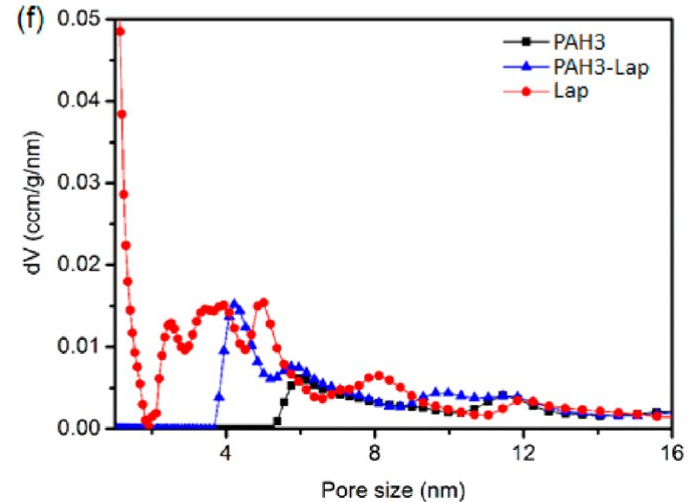

Figure 4. Surface and mechanical properties of hydrogels. (a) Schematic representation of PAH3-Lap hydrogel preparation. (b) Storage ( $\left.G^{\prime}\right)$ and loss $\left(G^{\prime \prime}\right)$ moduli of PAH3 and Lap compared to PAH3-Lap hydrogels. (c) Time sweep rheographs displaying thioxotropic properties of PAH3 and PAH3-Lap hydrogels. (d) Optical image showing the robustness and self-healing capacity of PAH3-Lap hydrogels. (e) $\mathrm{N}_{2}$ sorption isotherms of PAH3 (square traces), Lap (circular traces), and PAH3-Lap (triangular traces) xerogels. (f) Cumulative pore volume for PAH3, Lap, and PAH3-Lap xerogels.

charged head groups. However, PAH3 displayed a much stronger affinity for the nanoclay to create a higher-ordered nanostructure, potentially attributable to an additional hydrogen bond contribution from the imidazolium side chain. These results demonstrate how by tuning the charged headgroup of PAs, it is possible to systematically optimize the supramolecular interactions between PAs and nanoclay nanomaterials, which will potentially determine the gelation kinetic and mechanical properties of the resulting PA-nanoclay hydrogels on a macroscale.
Fabrication of PAH3-Lap Nanocomposite Hydrogels. Having established the underlying PA-nanoclay coassembling mechanism, we then focused on synthesizing hydrogels using PAH3 and taking advantage of the modular nature of our material design. Given the unique chemistry of histidine, we prepared PAH3-Lap hydrogels by immersing PAH3 solution $(2 \% \mathrm{w} / \mathrm{v})$ into a large volume of Lap solution $(2.5 \% \mathrm{w} / \mathrm{v})$ exfoliated with the sodium salt of poly(acrylic acid) ( $\mathrm{Mw}=5$ $\mathrm{kDa}, 0.06 \% \mathrm{w} / \mathrm{v}$ ). Within $30 \mathrm{~min}$ of immersion, self-supported hydrogels were formed in the Lap solution and the hydrogel was about the size of the PAH3 droplet $(\sim 10 \mathrm{~mm})$, suggesting 
that the gelation was driven by a diffusion mechanism whereby Lap diffuses into the droplet of PAH3 to trigger PAH3-Lap coassembly, which then leads to an entangled network of PAH3 nanofibers and Lap nanodisks (Figure 4a). In contrast, immersion of Lap into PAH3 did not produce stable hydrogels, which may result from a rapid diffusion of PAH3 toward the Lap nanodisk suspension and inability to concentrate two components in a compartmentalized fashion. Also, when Lap was used without exfoliation, partial hydrogels were formed which might be attributed to electrostatic repulsion between the positive edges of Lap disks and the cationic imidazole side chain of PAH3 or inhomogeneous dispersion of Lap in an aqueous medium. In contrast, the lysine-based analogue (PAK3; $\mathrm{CH}_{3}-\left(\mathrm{CH}_{2}\right)_{14}-\mathrm{CONH}-$ VVVAAAKKK-CONH${ }_{2}$ ) only formed weak hydrogels while the glutamic acid-based analogue (PAE3; $\mathrm{CH}_{3}-\left(\mathrm{CH}_{2}\right)_{14^{-}}$ CONH-VVVAAAEEE-CONH${ }_{2}$ ) did not cause gelation, suggesting that the presence of histidine aromatic imidazole head groups is key to preparing stable and strong hydrogels.

Characterization of Mechanical and Surface Properties of PAH3-Lap Hydrogels. Application of Dynamic Rheometry to Characterize the Viscoelastic Properties of PA-Lap. In order to assess the impact of Lap on the mechanical properties of PAH3 hydrogels, we used dynamic oscillatory rheology to measure the storage $\left(G^{\prime}\right)$ and loss $\left(G^{\prime \prime}\right)$ moduli. $G^{\prime}$ and $G^{\prime \prime}$ of PAH3-Lap hydrogels were frequency independent, with $G^{\prime}$ dominating $G^{\prime \prime}$ across the whole range of frequencies tested $(0.1-50 \mathrm{~Hz})$ and at constant strain $\gamma(0.5 \%)$ (Supporting Information Figure S3). These results confirm a quasi-solid-like nature of PAH3-Lap hydrogels. The $G^{\prime}$ (70.89 $\pm 10.62 \mathrm{kPa})$ and $G^{\prime \prime}(10.54 \pm 2.11 \mathrm{kPa})$ values for PAH3-Lap nanocomposite hydrogels were greater than the $G^{\prime}(10 \pm 0.51$ $\mathrm{kPa})$ and $G^{\prime \prime}(1 \pm 0.09 \mathrm{kPa})$ values of PAH3 hydrogels (Figure 4b). To further confirm that supramolecular coassembly with Lap can improve the stiffness of other PA hydrogels, we prepared PAK3-Lap hydrogels. PAK3 is known to produce weak hydrogels $\left(G^{\prime} \sim 1 \mathrm{kPa}\right)$ by charge screening. Here, we observed that PAK3-Lap hydrogels displayed a $G^{\prime}$ of $\sim 10 \mathrm{kPa}$ (Supporting Information Figure S4), which is significantly lower than that of PAH3-Lap hydrogels $(\sim 70.89 \mathrm{kPa})$. This enhanced stiffness of PAH3-Lap over PAK3-Lap is expected as the aromatic imidazole side chain of the histidine residue is known to play a critical role in promoting the self-assembly of proteinaceous fibers leading to self-healable and mechanically reinforced spider fangs, sandworm jaws, or mussel byssals. ${ }^{47}$ Therefore, we reasoned that the aromatic side chain of histidine might provide additional noncovalent interactions, making the surface free energy of adsorption $(\varepsilon)$ of PAH3 to Lap nanodisks greater or equal to the thermal energy $\left(\mathrm{K}_{\mathrm{B}} \mathrm{T}\right){ }^{52}$ This is in agreement with our initial speculation based on the molecular dynamic simulations data (Figure 3).

In addition, we carried out strain amplitude sweep measurements to determine the strain-to-break values of PAH3-Lap against PAH3. The results indicated that $G^{\prime}$ of PAH3-Lap decreased rapidly when subjected to a magnitude of strain beyond the critical strain value $(\gamma=6 \%)$. On the other hand, $G^{\prime}$ values of PAH3 hydrogels decreased rapidly at a much greater strain value $(\gamma=13 \%)$, suggesting that PAH3 hydrogels are more viscoelastic than PAH3-Lap hydrogels. Put together, the enhanced stiffnesses of PAH3-Lap over PAH3 and PAK3-Lap over PAK3 suggest that the 2D structure of Lap promotes a strong physical interaction between the PA nanofibers and Lap nanodisks (Figure 1b). It is noteworthy that while PAs offer a powerful platform to design precise and bioactive matrixes, these materials tend to suffer from poor mechanical properties $\left(G^{\prime}<10 \mathrm{kPa}\right)$, making our organicinorganic hybridization an attractive strategy to prepare another class of PA-based hydrogels with dramatically improved mechanical properties ( $\left.G_{\text {PA-Lap }}^{\prime} \sim 71 \mathrm{kPa}\right)$. This strategy has been demonstrated using Lap with other organic components such as silk $\left(G^{\prime}{ }_{\text {silk-Lap }} \sim 150 \mathrm{kPa}\right)^{37}$ and dendritic molecular binders $\left(G^{\prime}{ }_{\text {dendron-Lap }} \sim 250 \mathrm{kPa}\right){ }^{48}$

Hydrogels Display Thixotropic and Self-Recovery Properties. Dynamic amplitude measurements were subsequently carried out to investigate the self-recovery or thixotropic property of PAH3-Lap and PAH3 hydrogels following network rupture at high strain. We applied a high strain amplitude (100\%) to rupture the hydrogel networks followed by a low strain amplitude $(0.1 \%)$ to investigate the rate and extent of recovery of the hydrogels. Under the high strain amplitude (100\%), the hydrogels underwent internal breakage leading to a significant decrease in $G^{\prime}$ and inversion of $G^{\prime}$ and $G^{\prime \prime}$. The inversion signifies that the liquid-like behavior dominates the solid-like nature of the hydrogels. When the strain amplitude was reduced to $0.1 \%$, both PAH3 and PAH3Lap hydrogels displayed fast recovery within seconds (Figure 4c), making both types of hydrogels potentially injectable. While PAH3 hydrogels exhibited complete recovery to the same initial $G^{\prime}$, PAH3-Lap hydrogels exhibited enhanced recovery beyond the initial $G^{\prime}$ (from 60 to $100 \mathrm{kPa}$ ) after the first strain cycle (Figure $4 c$ green trace). Such enhanced recovery has previously been reported in self-assembling hydrogels, ${ }^{53,54}$ and we reasoned it is suggestive of structural reorganization of the hydrogels. Macroscopically, PAH3-Lap hydrogels were able to self-heal in air (Figure 4d). These results suggest that Lap enhanced stability and facilitates selfhealing in PAH3-Lap hydrogels. The rapid self-healing process exhibited by PAH3-Lap hydrogels may result largely from both the attachment of the imidazolium group of the PAH3 to the exfoliated Lap surfaces and the intrinsic propensity of PAH3 networks to rapidly recover after rupture.

Characterization of Surface Properties of PAH3-Lap Hydrogels. Having characterized the bulk properties of PAH3-Lap hydrogels, we then used a nitrogen gas adsorption method based on quenched solid density functional theory (QSDFT) to investigate the impact of PA-Lap coassembly on surface properties including pore volumes and pore diameters. The experiments were conducted on PAH3-Lap dried xerogels and compared to the individual components. In all cases, the xerogels exhibited a surface profile that is consistent with typeIII adsorption-desorption curves with distinct capillary condensation steps. The adsorption isotherms (volume of nitrogen per gram of materials at standard temperature and pressure (STP)) revealed that the surface areas of Lap and PAH3 xerogels were $165 \mathrm{~m}^{2} / \mathrm{g}$ and $18 \mathrm{~m}^{2} / \mathrm{g}$, respectively (Figure 4e, Supporting Information Figure S5). Upon coassembly, the surface area $\left(50 \mathrm{~m}^{2} / \mathrm{g}\right)$ of PAH3-Lap was higher than that of PAH3 xerogels, implying that the surface adsorption of PAH3 to Lap nanodisks considerably decreases the surface area of the Lap nanodisks. For Lap, a number of peaks which span between $0.25-0.78 \mathrm{~nm}$ (micropores), 2.00$6.00 \mathrm{~nm}$ (mesopores), and $12.00 \mathrm{~nm}$ (macropores) (Figure 4f_red trace) were observed, suggesting that Lap displays a hierarchical polymodal pore size distributions. ${ }^{55,56}$ In contrast, PAH3 xerogel pore size distribution profiles displayed a broad peak centered at $6 \mathrm{~nm}$ (mesopores) and another weak peak at 
(a)

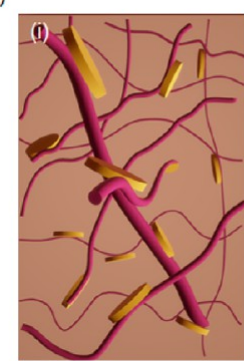

- $=\mathrm{Ca}^{2+}$

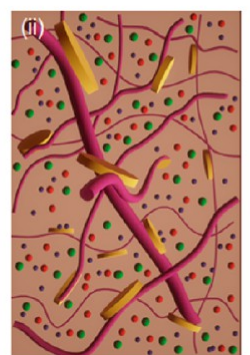

$\bullet=\mathrm{HPO}_{4}{ }^{3-}$

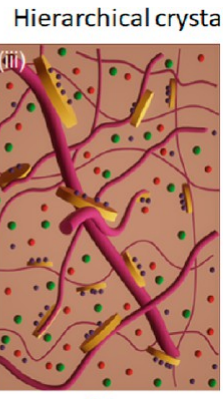

\% nuclei

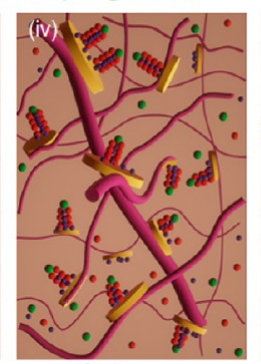

$>K=$ growing nanorods
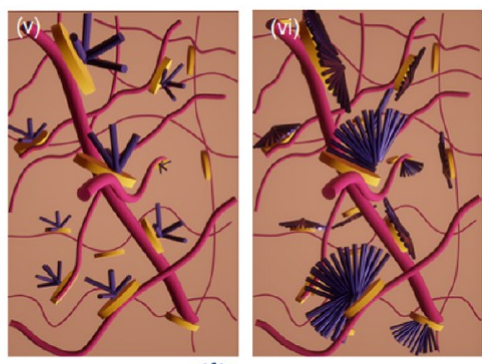

= spherical cluster
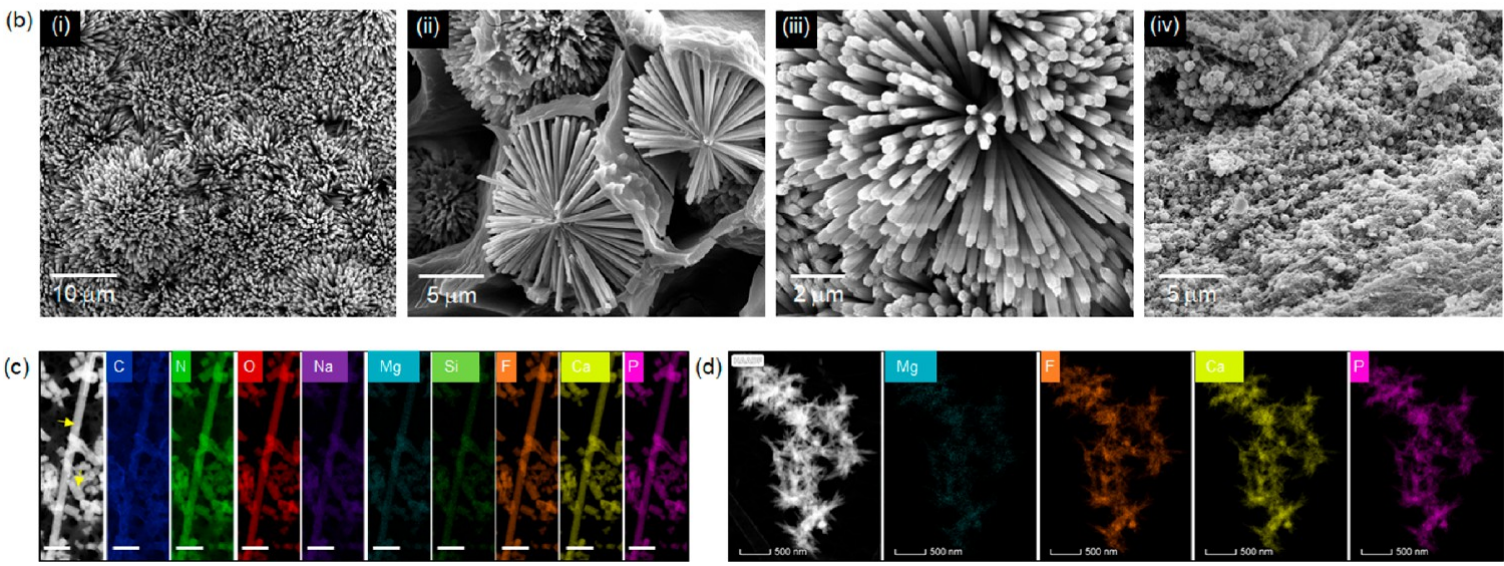

Figure 5. Biomineralization in hydrogels. (a) Schematic representation of the biomineralization process. PAH3-Lap hydrogel was immersed in a mineralizing bath (i) followed by a gradual diffusion of the ionic precursors into the hydrogel cavity (ii), which resulted in an initial electrostatic binding of calcium $\left(\mathrm{Ca}^{2+}\right)$ ions to the Lap surface (iii). Further association of phosphate $\left(\mathrm{HPO}_{4}{ }^{3-}\right)$ and fluoride $\left(\mathrm{F}^{-}\right)$ions with the Lap-Ca ${ }^{2+}$ complex produces nuclei (iv) that developed into oriented nanorods in a time-dependent manner ( $v$ ), which organize hierarchically into spherical clusters (vi). (b) Scanning electron micrographs showing (i) dense crystal formation on the surface of a PAH3Lap hydrogel, which are organized into (ii, iii) spherical clusters of nanorods within the cavity of the hydrogels after 8 days in mineralizing solution. Also, PAH3 hydrogels were mineralized but spherical and amorphous crystals were formed (iv). (c) HRTEM-EDX elemental mapping of nanorods formed in PAH3-Lap hydrogels after 8 days. Yellow arrows indicate fluoridated hydroxyapatite nanorods. Elemental mapping of the nanorods shows carbon C (blue), nitrogen $\mathrm{N}$ (green), oxygen $\mathrm{O}$ (red), sodium $\mathrm{Na}$ (purple), magnesium Mg (cyan), silicon Si (green), fluorine F (orange), calcium Ca (yellow), and phosphorus P (pink). Scale bar: $100 \mathrm{~nm}$. This was contrasted with the morphology of the (d) needle-like crystals that formed in PAH3-Lap hydrogels within $2 \mathrm{~h}$ of incubation in mineralizing solutions.

$12 \mathrm{~nm}$, indicating that PAH3 xerogels exhibited a uniform pore size distribution (Figure $4 \mathrm{f}$ _black trace). It is important to note that the observed porosity profile for PAH3 xerogels could be due to lyophilization of the gels prior to analysis. The pore size distribution curves for PAH3-Lap showed multiple peaks centered at 4.2, 6.0, 10.0, and $12.0 \mathrm{~nm}$. The peaks at 4.2 and $6.0 \mathrm{~nm}$ are characteristic fingerprints of Lap and PAH3 xerogels, respectively (Figure 4f_blue trace), which confirm the heterogeneity of the PAH3-Lap internal structure. In contrast, the peaks corresponding to the micropores of Lap are not apparent in PAH3-Lap, which suggests that surface adsorption of the PAH3 nanofibers to the Lap disk removes access to the micropores by blocking them. We therefore hypothesized that the molecular diversity and heterogeneous functional groups of PAH3-Lap hydrogels in relation to PAH3 hydrogels may provide an opportunity to nucleate and grow apatite crystals within the confined $3 \mathrm{D}$ framework of the hydrogels.

PAH3-Lap Hydrogels to Guide In Situ Mineralization. Features That Make Organic-Inorganic Hydrogels an Ideal Model for Biomineralization. Hydrogels have been harnessed as structural frameworks to elucidate the origins of biological control over crystal morphology, orientation, and matrix incorporation. ${ }^{3}$ These materials display (i) volumetric confine- ment to control crystal growth, (ii) nanoporosity to control diffusion rates, capacity to tune concentrations and supersaturation of solutes, and (iii) internal nanostructures with high surface area to template crystal growth., ${ }^{3,57}$ Unlike the classical mechanism of atom or molecule mediated growth of single crystals, the particle mediated growth and assembly mechanisms leading to the formation of single crystals have been recognized as emerging nonclassical biomineralization processes. ${ }^{58}$ This phenomenon is believed to result from both the free-energy landscapes and reaction dynamics that govern particle-particle interactions. ${ }^{59-61}$ Such reaction dynamics might account for the impressive mineral deposition recently observed by Paul and co-workers using DNA-Laponite hybrid hydrogel coatings on bone allografts. ${ }^{36}$ Therefore, we hypothesized that the surface area, functional groups, and structural anisotropy of Lap nanodisks can be harnessed in PAH3-Lap hydrogels to control the energy landscape at the substrate-nuclei interface during biomineralization in a timedependent manner, leading to the formation of multiinorganic-organic nano-objects.

To test this hypothesis, we used a known mineralizing solution to nucleate and trigger the growth of fluoridated hydroxyapatite nanocrystals. ${ }^{10}$ The PAH3-Lap hydrogels were submerged in the mineralizing solution $(20 \mathrm{~mL})$ and kept at 37 
(a)

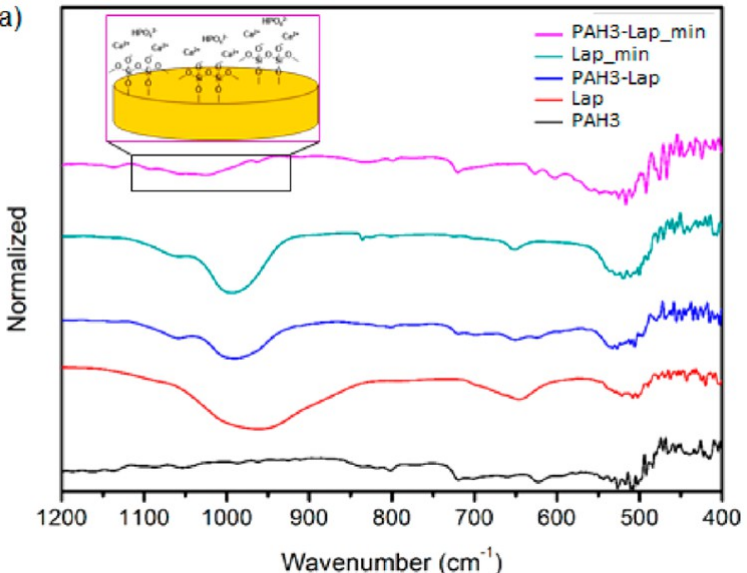

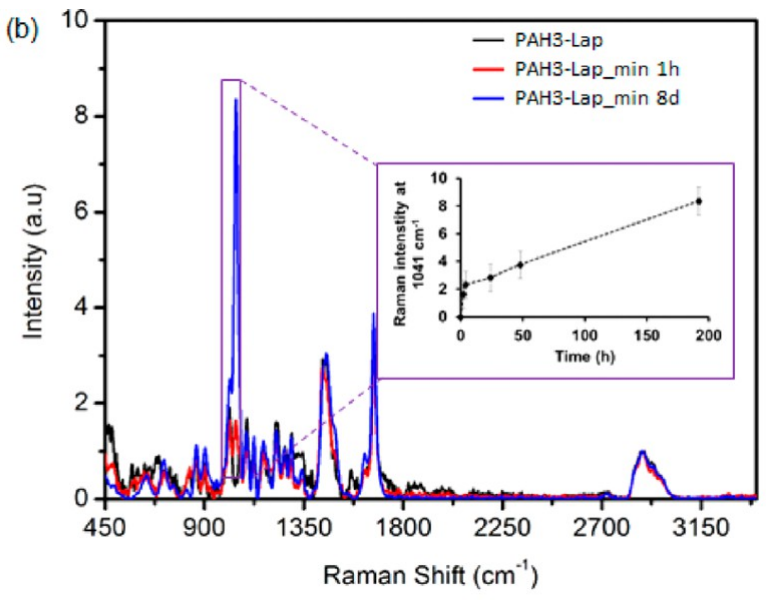

(c)

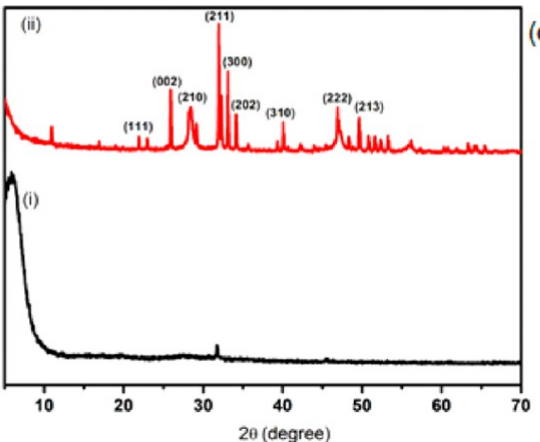

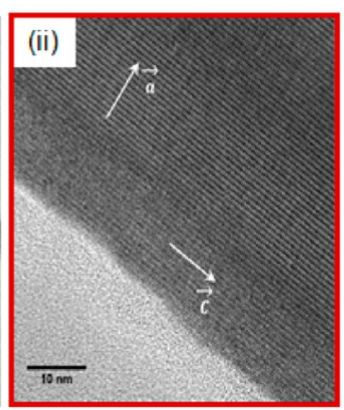

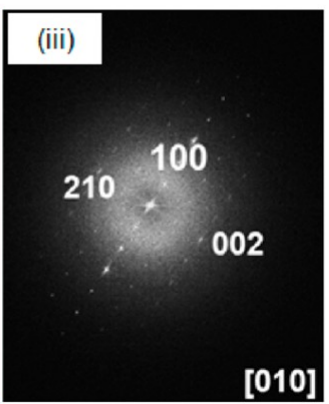

Figure 6. Characterization of biomineralization. (a) FTIR spectra indicating Lap silica oxide layer participation in biomineralization. (b) Normalized Raman spectra of unmineralized PAH3-Lap hydrogels and PAH3-Lap mineralized for 1 and 8 days. The inset is a plot of 1047 $\mathrm{cm}^{-1}$ signal intensity versus mineralization time. (c) X-ray powder diffraction (XRD) crystallographic profiles of mineralized (red trace) and unmineralized (black trace) PAH3-Lap hydrogels after 8 days of incubation in mineralizing solution. (d) (i) HRTEM image of hydroxyapatite nanorods formed in PAH3-Lap hydrogels, (ii) enlarged selected area (red square) of HRTEM image of nanorods, and (iii) FFT patterns of crystals viewed from the [010] crystallographic direction.

${ }^{\circ} \mathrm{C}$ (Figure 5a). We observed that the transparent PAH3-Lap hydrogels became cloudy within 8 days of incubation (Supporting Information Figure S6), suggesting its high mineralization capacity. In contrast, PAH3 hydrogels remained less opaque, indicating less mineralization than PAH3-Lap hydrogels. In order to confirm that this enhanced mineralization was due to the presence of Lap, we added Lap solution alone to the mineralizing solution and investigated crystal formation. Interestingly, Lap suspension exhibited white precipitates after 8 days of incubation, suggesting that Lap is able to drive nucleation and growth of apatite crystals. These results are potentially consistent with the ability of Lap nanodisks to act as catalysts for the formation of mineralized matrixes both in in vitro and in vivo. ${ }^{32-34}$ Similarly, silica hydrogels have previously been used to drive the formation of hematite $\left(\alpha \mathrm{Fe}_{2} \mathrm{O}_{3}\right)$ into hierarchical mosaic crystals displaying hierarchical structures inaccessible in solution-grown controls, indicating that silicate materials display functionalities that promote heterogeneous nucleation and growth of crystals. ${ }^{62}$

Lap Nanodisks Are Essential for Nanorod Formation. Scanning electron microscopy (SEM) was used to examine the mineralization within the hydrogels. SEM micrographs of PAH3-Lap xerogels revealed the presence of high-aspect ratio apatite nanorod crystals ( $\sim 50 \mathrm{~nm}$ in cross-sectional diameter) on the surface of the mineralized hydrogels after 8 days in the mineralizing solution (Figure $5 \mathrm{~b}$ i). These apatite nanorods were organized hierarchically into well-defined microscopic clusters, which resemble mesocrystals. ${ }^{63}$ The clusters grew symmetrically and to similar sizes up to microns in diameter in "confined pockets" within the hydrogels (Figure 5b ii-iii). We hypothesize that these cluster structures are formed by the diffusion of ionic mineralization precursors through the PAH3Lap hydrogel, random nucleation across the internal walls of the hydrogel, and subsequent symmetric growth of apatite nanorods along the precursor-crystal interface (Figure 5c). This process of crystal growth and entrapment within integrated hybrid materials has been regarded as nanoscale incarceration by Mann. ${ }^{17}$ In contrast to this nanorod and microcluster organization within PAH3-Lap hydrogels, we observed spherical nanocrystals (diameter $\sim 50 \mathrm{~nm}$ ) in PAH3 hydrogels, which are reminiscent of previous studies by Stupp and colleagues. ${ }^{64}$ Based on these results, we propose that the integrated nanofibers and nanodisks within PAH3-Lap hydrogels provide a $3 \mathrm{D}$ organic-inorganic framework of heterogeneous nucleation sites for hierarchical mineralization. To explore the possibility that Lap is acting as a catalyst for mineralization in the PAH3-Lap hydrogels, we hybridized Lap with PAK3 knowing that PAK3 does not induce mineralization of apatite in its own right. In this case, we again observed formation of both nanorods and nanospheres within the hydrogels after 8 days of incubation (Supporting Information Figure S7), thus suggesting that the presence of Lap nanodisks in PA-based hydrogels played a key role in the nucleation and growth of crystals within the hydrogels. 
Elemental Mapping to Elucidate Colocalization of Lap and Hydroxyapatite. Given the hierarchical nanorod-cluster mineralization within PAH3-Lap, we then investigated nanorod crystal formation in further detail. First, to verify interactions between Lap nanodisks and the mineralization ionic precursors, we used HRTEM-EDS to map the elemental composition of the mineralized PAH3-Lap hydrogels. HRTEM images confirmed the formation of the $\sim 50 \mathrm{~nm}$ diameter hexagonal nanorod crystals in the PAH3-Lap hydrogels after 8 days of incubation (Figure 5c). Also, the HRTEM-EDS mapping revealed colocalization of carbon (C), nitrogen $(\mathrm{N})$, oxygen $(\mathrm{O})$, sodium $(\mathrm{Na})$, magnesium $(\mathrm{Mg})$, silicon $(\mathrm{Si})$, fluoride $(\mathrm{F})$, calcium $(\mathrm{Ca})$, and phosphorus $(\mathrm{P})$ along the nanorods, which suggests the incorporation of dissolved PAH3 and Lap into the nanocrystals during growth. To gain insight into the early stage of this mineralization phenomenon, we examined the morphology and elemental composition of the crystals obtained after a $2 \mathrm{~h}$ incubation period in the mineralizing solution. HRTEM-EDS micrographs of PAH3-Lap hydrogels following a $2 \mathrm{~h}$ incubation period revealed an outward growth of the spherical clusters comprising the nanorods with colocalized elemental components of PAH3-Lap hydrogels (Figure 5d). Moreover, the nanorods appeared to be growing in the direction of the PAH3-Lap hydrogel nanofibers (Supporting Information Figure S8a), which suggests that the orientation of the nanofiber-nanodisk hybrid might be playing a key role in directing the hierarchical nanorod growth. Also, the white particles that sediment in the Lap solution were analyzed using HRTEM-EDS, which revealed the formation of agglomerated nanorods with elemental mapping showing both Lap characteristic elements and ionic precursors for mineralization (Supporting Information Figure S9). These results indicate that Lap might be serving as an essential template for nanorod growth within the organic-inorganic hydrogels due to its $2 \mathrm{D}$ ultrathin structure and surface chemistry.

FTIR Confirms Hydrogen Bond-Driven Interactions between Lap and Biominerals. Using Fourier transform infrared (FTIR) spectroscopy, we then investigated the mechanism of interaction between Lap and the ionic precursors present in the mineralizing solution. With the technique we also attempted to verify the identity of the apatite nanorods. According to the FTIR spectra (Figure 6a), the band at $970 \mathrm{~cm}^{-1}$ corresponds to $\mathrm{Si}-\mathrm{O}-\mathrm{Si}$ of Lap. This band shifts from 970 to $985 \mathrm{~cm}^{-1}$ in PAH3-Lap hydrogels, which suggests hydrogen-bonding interactions between PAH3 and Lap. Such red-shift in the $\mathrm{Si}-\mathrm{O}-\mathrm{Si}$ band of Lap has previously been observed in polymer-Lap composite hydrogels. ${ }^{65}$ After incubating PAH3-Lap in the mineralizing media for 8 days, the band became broader and was further shifted to a higher frequency $\left(c a .1022 \mathrm{~cm}^{-1}\right)$. Thus, we hypothesized that the mineralized PAH3-Lap hydrogels interacted noncovalently with the $\mathrm{Si}-\mathrm{OH}$ layer of Lap. To verify this, we incubated a Lap suspension in the mineralizing media under the same conditions for 8 days. The band of $\mathrm{Si}-\mathrm{OH}$ shifted from 970 to $995 \mathrm{~cm}^{-1}$, confirming Lap as an active catalyst for mineralization in PAH3-Lap hydrogels.

Time-Resolved Evolution of Nanocrystals and Associated Fingerprints to Understand Mechanisms of Biomineralization. Given the distinctive functional groups of Lap, PAH3, and the mineralized nanorods, Raman spectroscopy was used to elucidate their molecular composition within mineralized PAH3-Lap hydrogels. Furthermore, by taking advantage of the fingerprints of phosphate functional groups on the nanorods, we monitored the kinetics of crystal growth in PAH3-Lap hydrogels. The Raman spectra of the PAH3-Lap hydrogels mineralized for 8 days revealed vibrational frequencies corresponding to the internal $\mathrm{PO}_{4}{ }^{3-}$ mode. The vibrational frequencies of the $\mathrm{PO}_{4}{ }^{3-}$ were found to be $\nu 1=960 \mathrm{~cm}^{-1}$ and $\nu 3=1047 \mathrm{~cm}^{-1}$ (Figure $6 \mathrm{~b}$ ). These frequencies correspond to the characteristic symmetric $\mathrm{P}-\mathrm{O}$ stretching modes and the triply degenerate asymmetric $\mathrm{P}-\mathrm{O}$ stretching modes, respectively. ${ }^{66}$ Peaks at $1450 \mathrm{~cm}^{-1}(\mathrm{C}=\mathrm{C}$ stretch of the imidazole side chain) and $1675 \mathrm{~cm}^{-1}(\mathrm{C}=\mathrm{O}$ stretch, amide band $\mathrm{I})$ correspond to peptide vibrations from PAH3 while the peak at $1010 \mathrm{~cm}^{-1}$ corresponds to the $\mathrm{Si}-\mathrm{O}$ vibrational stretch from Lap present in the PAH3-Lap hydrogels. The amide band I at $1675 \mathrm{~cm}^{-1}$ further confirms the intrinsic $\beta$-sheet conformation of PAH3 nanofibers in the coassembled PAH3-Lap. ${ }^{67}$ By comparing this amide band I before and after mineralization, we observed no significant changes in the conformation of PAH3 nanofibers, thus suggesting that the PAH3 nanofibers maintained their spatial organization under the mineralization event.

We monitored the kinetics of crystal growth in the PAH3Lap hydrogels by observing the regions of the Raman spectra corresponding to the triply degenerate asymmetric $\mathrm{P}-\mathrm{O}$ stretching modes $\left(\nu 3=1047 \mathrm{~cm}^{-1}\right)$. At time $t=0$ (before mineralization), no Raman peak was apparent in this region. After mineralization for $1 \mathrm{~h}$, there was an emergence of the $\mathrm{P}-$ $\mathrm{O}$ stretching mode that featured two sharp peaks at 1005 and $1047 \mathrm{~cm}^{-1}$ (Figure 6b, Supporting Information Figure S10). The relative intensity of the $1047 \mathrm{~cm}^{-1}$ peak signal (all spectra were first normalized with respect to the $\mathrm{C}-\mathrm{H}$ signal intensity at $2800-3000 \mathrm{~cm}^{-1}$ ) increased rapidly within $4 \mathrm{~h}$ of mineralization and steadily afterward until the 8-day timepoint (Figure 6b_inset, Supporting Information Figure S10), indicating a two-phase crystal growth. Elemental analysis of the two stages of crystal growth revealed a $\mathrm{Ca} / \mathrm{P}$ ratio of 1.45 and 1.65 for the nanorods obtained at 4-h and 8-day time-points, respectively (Supporting Information Figure S8d). The former $\mathrm{Ca} / \mathrm{P}$ ratio is indicative of an amorphous calcium phosphate while the latter is characteristic of a hydroxyapatite crystal. Thus, the two-stage crystallization events exhibited an initial amorphous precursor phase, which steadily underwent a slow interaction with the mineralization ionic species diffused into the PAH3-Lap hydrogels, leading to a linear rate of growth to attain the hydroxyapatite composition with $\mathrm{Ca} / \mathrm{P}$ ratio 1.65 . Similarly, Raman spectra for PAH3 hydrogels mineralized for 8 days also displayed the key $\mathrm{PO}_{4}{ }^{3-}$ fingerprints $\left(\nu 1=960 \mathrm{~cm}^{-1}\right.$, $\nu 3=1047 \mathrm{~cm}^{-1}$ ) of hydroxyapatite formed in PAH3-Lap hydrogels as well as a Raman peak at $564 \mathrm{~cm}^{-1}$ (Figure 6b), which corresponds to the $\nu 4$ bending mode characteristic of $\mathrm{PO}_{4}{ }^{3-}$ in amorphous calcium phosphate. ${ }^{67} \mathrm{We}$ also used elemental analysis to show that the $\mathrm{Ca} / \mathrm{P}$ ratio is 1.1 for the amorphous calcium phosphate formed in the PAH3 hydrogels (Supporting Information Figure S12).

$X R D$ and Other Physical Analysis Techniques Confirm the Crystallographic Direction of Crystal Growth in the PAH3Lap Hydrogels. The X-ray diffraction pattern of the mineralized PAH3-Lap hydrogels compared to the unmineralized PAH3-Lap hydrogels indicated that the nanocrystals formed after 8 days were crystalline (Figure $6 \mathrm{c}$ ). More so, the diffraction peaks $(002)$ at $2 \theta=25.8^{\circ},(211)$ at $2 \theta=31.8^{\circ}$, (300) at $2 \theta=32.8^{\circ},(202)$ at $2 \theta=34.2^{\circ}$, and (222) at $2 \theta=$ $46.9^{\circ}$ (Figure 6c_ii) are consistent with the peaks for fluoridate 
a)

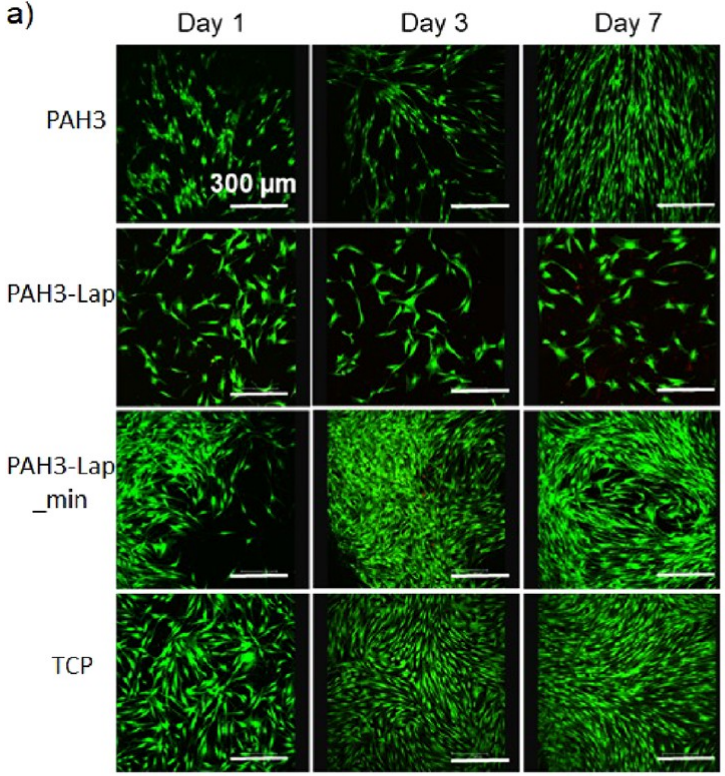

c)

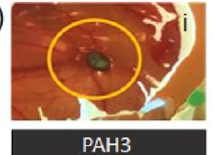

d)

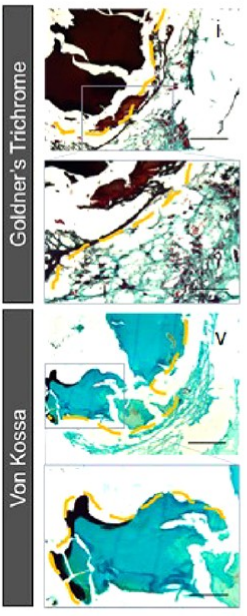

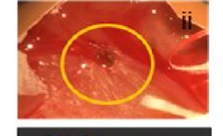

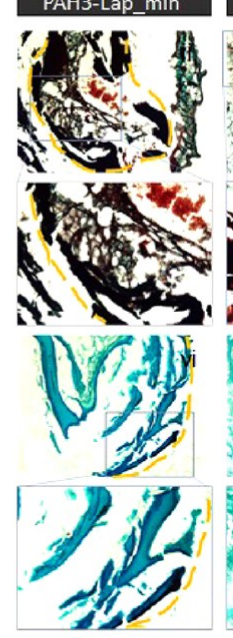

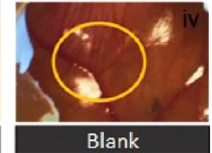

PAH3-Lap unmin

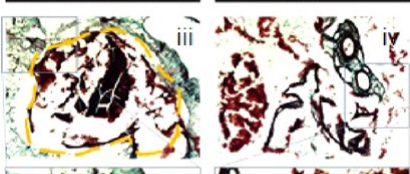

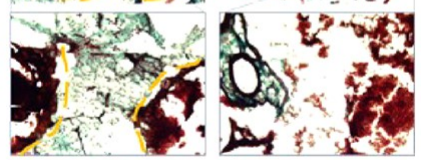
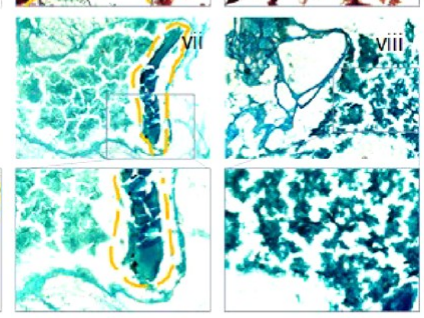

b)

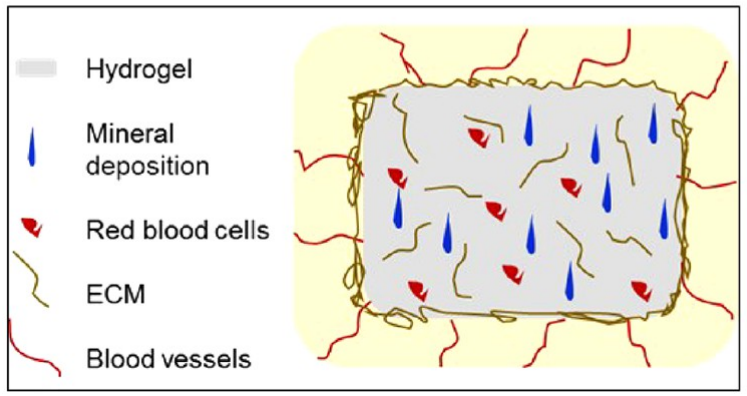

e)

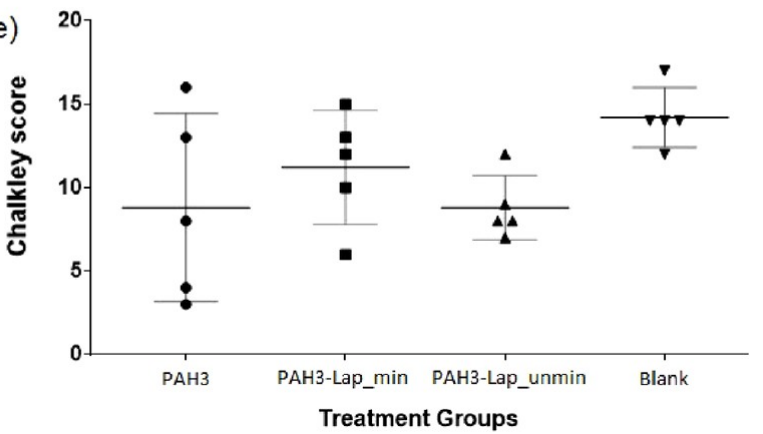

Figure 7. Biological applicability of PAH3-Lap hydrogels. (a) The in vitro applicability of the hydrogels was assessed by a LIVE/DEAD assay to test cell viability and proliferation of hBMSC on the hydrogels. The results revealed that cell viability and proliferation on mineralized PAH3-Lap hydrogels are more than those of cells growing on tissue culture plastic (TCP) for 7 days. (b) Schematics of CAM implantation of hydrogels. Hydrogels promote mineral deposition, red blood cell infiltration, and ECM and blood vessels formation. (c) Optical image of CAM implanted hydrogels. (i) PAH3 hydrogels implanted in CAM were surrounded by blood vessels in close proximity of the chorioallantoic membrane. Both (ii) PAH3-Lap and (iii) PAH3-Lap_min hydrogels were highly integrated with the chorioallatoic membrane with blood vessels penetrating the implanted hydrogels. (d) Histological analysis of CAM implanted hydrogels. Mineral deposition was found limited to the outer region of the implanted PAH3 hydrogels. Limited mineral deposition was observed in proximity of the PAH3-Lap hydrogel-membrane interface. Mineral deposition was found extensively within the PAH3-Lap.min hydrogels. Both PAH3-Lap and PAH3Lap_min hydrogels were extensively invaded by red blood cells. Blank samples were extensively penetrated by blood vessels but no mineral deposition. (e) Chalkley score of PAH3, PAH3-Lap, PAH3-Lap_min, and blank controls samples. Statistical significances were assessed by one-way ANOVA. Mean \pm SD $n=5$. Scale bar for $(\mathrm{d})=100 \mu \mathrm{m}$.

hydroxyapatite. ${ }^{57}$ Furthermore, the sharp 002 peak indicated that the nanorods were oriented along the $c$ axes, which is reminiscent of nanocrystal growth in both dental enamel and bone. ${ }^{68}$ A closer look at the HRTEM images of the mineralized PAH3-Lap hydrogels confirmed that the nanorods assumed a preferred orientation along the $c$ axes of the fluoridated hydroxyapatite (Figure 6d_i-ii). Also, the fast Fourier transform (FFT) patterns viewed from the [010] crystallographic direction are consistent with the XRD data and HRTEM images, indicating that the crystal lattices were only observed in the nanorods with long axes along the direction corresponding to the reflection area (Figure $6 \mathrm{~d}$ _iii). These results further confirm that the $c$ axes of the crystal lattices were mainly aligned along the long axes of the nanorods. Although, the potential of Lap to trigger cascades of cell signaling that mediate bone formation in vivo is well-known, ${ }^{28}$ these results showcase the potential of Lap nanodisks as efficient templates to guide nanocrystal growth via a nonclassical particle attachment mechanism and in a hierarchical manner.

Evaluation of Biocompatibility of Mineralized PAH3Lap Hydrogels. The biological relevance of PAH3 as well as mineralized and unmineralized PAH3-Lap hydrogels as functional biomaterials was assessed in vitro by seeding human bone marrow stromal cells (hBMSCs) on the hydrogels. As shown in Figure 7a, live skeletal cells stained with calcein AM were predominantly visible on the hydrogels after 7 days in culture, indicating excellent cytocompatibility across the hydrogels. However, hBMSCs proliferated significantly more on the mineralized PAH3-Lap hydrogels compared to the PAH3 and unmineralized PAH3-Lap hydrogels, as well as on tissue culture plastic (TCP) (Supporting Information Figure S13). To further assess the biological functionality of the hydrogels ex vivo, we used the 
chorioallantoic membrane (CAM) assay of the chick embryo, to examine tissue integration and blood vessel and extracellular matrix formation as previously published. ${ }^{69}$ Histological analysis of the implanted PAH3 and unmineralized and mineralized PAH3-Lap hydrogels after 7 days demonstrated that the hydrogels fully integrated within the CAM (Figure $7 \mathrm{c}$ ii). However, while blood vessels were only visible on the surface of the PAH3 hydrogels (Figure 7c_i), both unmineralized (Figure $7 c$ _iii) and mineralized (Figure $7 c$ _ii) PAH3-Lap hydrogels exhibited blood vessels growing within (Figure 7c_ii-iii), indicating a higher capacity of neovascularization. Using Goldner's and von Kossa staining, we confirmed extensive mineral deposition in the mineralized PAH3-Lap (PAH3-Lap_min) hydrogels (Figure $7 \mathrm{~d}$ ii,vi) in comparison to unmineralized hydrogels (Figure $7 \mathrm{c}$ - iii,vii). No mineral deposition was apparent in the blank eggs (Figure 7d_iv,viii). Similar to the blank samples, both PAH3-Lap (Figure 7d iii) and PAH3-Lap-min (Figure 7d ii) hydrogels were extensively invaded by red blood cells. The Chalkley score (Figure 7e) shows there is no significant difference between the level of vascularization in the treatment groups and the controls. These results suggest that the PAH3-Lap hydrogels can serve as robust multifunctional matrices with the capacity to promote cell growth, trigger hierarchical mineralization and bone tissue formation, and promote vascularization.

\section{CONCLUSION}

We have developed a coassembling organic-inorganic hydrogel platform for in vitro crystal growth mediated by a particle attachment mechanism within a 3D supramolecular confined framework. The design strategy hinges on electrostatic interactions between Lap nanodisks and cationic PAH3 molecules to integrate the intrinsic properties of the organic and inorganic components into distinctive organic-inorganic hydrogel structures. The resulting materials displayed high surface area, high mechanical properties, and self-healing properties. Furthermore, the coassembling PA-Lap hydrogel displayed a nanoscale architecture that served as confined spaces for the hierarchical growth of hydroxyapatite from ordered nanorods into well-defined spherical clusters. The study explores this mineralization mechanism as a biomimetic 3D model to modulate nucleation and spatiotemporal organization of fluoridated hydroxyapatite. This model was used to understand the role of both Lap nanodisks and PAH3 nanofibers within PAH3-Lap hydrogels in guiding the growth of the hydroxyapatite nanorods across multiple length scales. At the atomic level, the mineralization of PAH3-Lap depended on a diffusion-driven process where local ionic concentration and supersaturation are mediated by supramolecular interactions with Lap. Furthermore, the nanoscale architecture of the PAH3-Lap hydrogels facilitated incarceration of the nanorod crystals and subsequent growth into the distinctive spherical clusters at the microscale. Interestingly, these mineralized PAH3-Lap nanocomposite hydrogels outperformed all control groups in supporting cell growth, stimulation of cell ingress, blood vessel infiltration, ECM production, and mineral deposition in a CAM model. In addition to these advantages, the shear-thinning property of the system makes it a suitable material to serve as a bioink for 3D printing applications. Overall, this study presents a nanotechnology approach to the design of integrated and higher-ordered self-assembling nanomaterials with potential widespread applications in regenerative medicine.

\section{EXPERIMENTAL METHODS}

Zeta Potential $(\zeta)$. All $\zeta$-potential measurements were performed after resuspension of the PAs at a concentration of $0.1 \% \mathrm{w} / \mathrm{v}$ in ultrapure water. After loading the samples into folded capillary cells, measurements were performed at $25{ }^{\circ} \mathrm{C}$ using a $\zeta$-sizer instrument (Nano-ZS Zen 3600, Malvern Instruments, UK). For each PA, three separate samples were measured with at least five runs per sample.

Circular Dichroism Spectroscopy. Circular dichroism (CD) was measured with a Chirascan circular dichroism spectrometer (Applied Photophysics Limited, UK) using a quartz cell with a $1 \mathrm{~mm}$ path length and the following parameters: data pitch, $0.5 \mathrm{~nm}$; scanning mode, continuous; scanning speed, $100 \mathrm{~nm} / \mathrm{min}$; bandwidth, $2 \mathrm{~nm}$; accumulation, 5. All CD data are presented as ellipticity and recorded in millidegree (mdeg). CD measurements were performed on aqueous solutions of PAH3 ( $0.1 \% \mathrm{w} / \mathrm{v})$, Lap $(0.25 \%)$, and their mixtures. CD spectra were obtained by signal integrating 3 scans, from 190 to $260 \mathrm{~nm}$ at a speed of $50 \mathrm{~nm} / \mathrm{min}$. Data were processed by a simple moving average and smoothing method.

Small-Angle Neutron Scattering (SANS) Analysis of Hydrogel Nanostructures. Synchrotron small-angle neutron scattering (SANS) measurements were performed on the fixed-geometry, timeof-flight LOQ diffractometer (ISIS Neutron and Muon Source, Oxfordshire, UK). A white beam of radiation with neutron wavelengths spanning 2.2 to $10 \AA$ enabled access to a $Q[Q=4 \pi$ $\sin (\theta / 2) / \lambda]$ range of 0.004 to $0.4 \AA^{-1}$ with a fixed-sample detector distance of $4.1 \mathrm{~m}$. The cuvettes were mounted in aluminum holders. The time taken for each measurement was approximately $30 \mathrm{~min}$. All scattering data were normalized for the sample transmission, the backgrounds were corrected using a quartz cell filled with $\mathrm{D}_{2} \mathrm{O}$, and the linearity and efficiency of the detector response were corrected using the instrument-specific software.

Atomic Force Microscopy (AFM). AFM was performed on a Bruker Multimode 8 AFM with a Nanoscope V controller using PeakForce Tapping mode with a ScanAsyst Air cantilever (spring constant $0.4 \mathrm{~N} / \mathrm{m}$ ). The cantilever was calibrated using the automated "no touch" calibration routine built into the software. Solutions of PAH3 (0.01\% w/v, $40 \mu \mathrm{L})$, Lap $(0.025 \% \mathrm{w} / \mathrm{v}, 40 \mu \mathrm{L})$, and PAH3/ Lap mixtures were dropped onto freshly cleaved mica surfaces. The samples were air-dried at room temperature for $24 \mathrm{~h}$ and imaged with a PeakForce set point of $500 \mathrm{pN}$ with a PeakForce amplitude of 30 $\mathrm{nm}$ and frequency of $4 \mathrm{kHz}$. Images were acquired at $512 \times 512$ pixels at a line rate of $2.8 \mathrm{~Hz}$. The height images were processed in the Nanoscope Analysis software after using first order flattening to remove tilt. Images were processed in Nanoscope 1.7.

Transmission Electron Microscopy (TEM) and High-Resolution TEM (HRTEM). Aqueous solutions of PAH3 (0.01\% w/v) and Lap $(0.025 \% \mathrm{w} / \mathrm{v}$, exfoliated with $0.0068 \% \mathrm{w} / \mathrm{v}$ ASAP) were dissolved in ultrapure water. Similarly, mixtures of PAH3 $(0.02 \% \mathrm{w} / \mathrm{v})$ and Lap $(0.5 \mathrm{wt} \% / \mathrm{v})$ were also prepared. Samples were mounted on a copper TEM plasma etched holey carbon-coated copper grid (Agar Scientific, Stansted, UK). The grids were immersed in the sample solutions for 5 min. Excess was removed on filter paper before incubation with $2 \%$ uranylacetate solution for $30 \mathrm{~s}$. Grids were then washed with ultrapure water for $30 \mathrm{~s}$ and air-dried for $24 \mathrm{~h}$ at room temperature. Bright-field TEM imaging was performed on a JEOL 1230 transmission electron microscope operated at an acceleration voltage of $80 \mathrm{kV}$. All the images were recorded by a Morada CCD camera (Image Systems). At least three images were taken per sample for further analysis. Highresolution transmission electron microscope (HRTEM) images, selected area electron diffraction (SAED) patterns, scanning transmission electron microscope (STEM) images, and energy dispersive $\mathrm{X}$-ray spectroscopy (EDS) spectrum images were obtained with a FEI Talos F200X microscope equipped with an X-FEG electron source and Super-X SDD EDS detectors. The experiment was performed using an acceleration voltage of $200 \mathrm{kV}$ and a beam current of 
approximately $1 \mathrm{nA}$. TEM images were recorded with a FEI CETA $4 \mathrm{k}$ $\mathrm{x} 4 \mathrm{k}$ CMOS camera. STEM images were acquired with HAADF and BF detectors.

Preparation of Hydrogels. An aqueous solution of Lap (2.5\% $\mathrm{w} / \mathrm{v}$ ) was prepared by adding the requisite amount of Lap powder to a stirred suspension of ASAP $(0.06 \% \mathrm{w} / \mathrm{v})$ in ultrapure water. The Lap suspension was sonicated for $30 \mathrm{~min}$ until a clear transparent sample was obtained. Aqueous solutions of PA $(2 \% \mathrm{w} / \mathrm{v})$ were prepared in HEPES buffer. PA-Lap hydrogels were prepared by injecting a solution of PA $(20 \mu \mathrm{L})$ into a larger volume of Lap $(100 \mu \mathrm{L})$. Gelation was allowed to proceed overnight at room temperature. Hydrogels of PAH3 (2\% w/v) were prepared by basifying an aqueous solution of PAH3 with $\mathrm{NaOH}(1 \mathrm{M})$.

Dynamic Rheological Measurements. Rheological measurements were performed using a Discovery Hybrid Rheometer, RheoDHR3 (TA Instruments). All data were collected at $25{ }^{\circ} \mathrm{C}$. The preformed hydrogels were added to the center of the bottom plate, and the top parallel plate (with $8 \mathrm{~mm}$ diameter) was lowered to a gap of $100 \mu \mathrm{m}$. The amplitude sweep measurements were performed between 0.1 and $50 \%$ strain at constant frequency $(1 \mathrm{~Hz})$. Similarly, frequency sweep rheographs were obtained between 0.1 and $20 \mathrm{~Hz}$ at constant strain (0.5\%). Self-healing was assessed initially at $0.1 \%$ strain for $100 \mathrm{~s}$, then at $100 \%$ strain for $200 \mathrm{~s}, 0.1 \%$ strain for $200 \mathrm{~s}$, $100 \%$ strain for $200 \mathrm{~s}$, and $0.1 \%$ strain for $400 \mathrm{~s}$.

Characterization of Surface Properties of Xerogels. Nitrogen sorption isotherms of the lyophilized xerogels were measured at $77 \mathrm{~K}$ using an Autosorb-IQ system (Quantachrome Instrument, USA). Before measurements, the samples were degassed in a vacuum at 120 ${ }^{\circ} \mathrm{C}$ overnight. The specific surface areas $\left(S_{\mathrm{BET}}\right)$ were calculated by the multipoint Brunauer-Emmet-Teller method using adsorption data in a relative pressure range from 0.04 to 0.2 , and the pore-size distribution was calculated based on quenched solid density function theory (QSDFT) using the adsorption branches of isotherms assuming slit and cylindrical pore geometries. By using the BarrettJoyner-Halenda $(\mathrm{BJH})$ model, the mesoporous surface areas $\left(\mathrm{S}_{\mathrm{BJH}}\right)$ were calculated from the adsorption line. The microporous surface areas $\left(\mathrm{S}_{\mathrm{DR}}\right)$ were calculated from the adsorption line by the DubininRadushkevich (DR) model.

Biomineralization of Hydrogels. The mineralizing solutions were prepared as previously reported by Elsharkawy et al. ${ }^{10}$ Briefly, an aqueous suspension of hydroxyapatite powder $(2 \mathrm{mM})$ and sodium fluoride $(2 \mathrm{mM})$ was prepared in deionized water with continuous stirring. Then, $69 \%$ nitric acid was added dropwise to the suspension to aid a complete dissolution of the hydroxyapatite precipitates at $\mathrm{pH}$ 2.4. Thereafter, an aqueous solution of ammonium hydroxide $(30 \%)$ was added dropwise to the hydroxyapatite solution until it reached $\mathrm{pH}$ 6. Various hydrogels were then immersed in the hydroxyapatite solutions and incubated for 8 days at $37{ }^{\circ} \mathrm{C}$ using a temperaturecontrolled incubator (LTE Scientific, Oldham, UK).

Monitoring of the Biomineralization Process by Raman Spectroscopy. All Raman analysis was carried out on a confocal WITEC Alpha300 system utilizing a $785 \mathrm{~nm}$ laser and a $20 \times$ (S Plan Fluor, NA 0.45, ELWD) objective lens. Raman scatter was collected in a backscattering geometry. A small amount of each sample was placed on a microscope glass slide which had been previously cleaned with a methanol-soaked tissue, with a new slide used for each sample. The incident laser power was constant for all samples at $63 \mathrm{~mW}$. No signal loss was observed, for example due to photobleaching or carbonization, when samples were irradiated on the same spot in triplicate with integration times ranging from 10 to $60 \mathrm{~s}$. All spectra processing was performed using SpectraGryph 1.2 involving (1) cosmic ray removal, (2) background correction, and then (3) subsequent normalization. An advanced baseline correction protocol available in the SpectroGryph software was applied which fits a polynomial curve to the spectral regions where there is no Raman peak and enables subtraction of the variable y-offset associated with the luminescence background. To enable comparison of the relative changes in the Raman intensity of the $1047 \mathrm{~cm}^{-1}$ peak in Figures 6 and S10, all spectra were normalized with respect to the peak intensity in the $2800-3000 \mathrm{~cm}^{-1}$ region. This approach was adopted as the integration time was varied between samples to optimize the signal-tonoise ratio alongside variation in background luminescence with mineralization times. However, the $\mathrm{C}-\mathrm{H}$ vibrational spectral shape across 2800-3000 $\mathrm{cm}^{-1}$ remained relatively unchanged for each sample, and the Raman peak intensity was also observed to change proportionally with integration time in this region. For each measurement, multiple spectra were acquired across the sample with the focus depth also optimized, which revealed good uniformity and ensured that the spectra presented are representative of the sample.

Synthesis and Purification of Peptide Amphiphiles. The peptide amphiphiles (PAs) were synthesized using solid-phase peptide synthesis (SPPS) on a Liberty Blue automated microwave peptide synthesizer (CEM, UK). The standard 9-fluorenylmethoxycarbonyl (Fmoc) protection chemistry on a 4-methylbenzhydrylamine (MBHA). Rink amide resin (Novabiochem Corporation, UK) was employed. PAs were purified using preparative high-performance liquid chromatography (Waters, USA) with a reverse-phase Xbridge C18 column (Waters, USA) and a water/acetonitrile $\left(0.1 \% \mathrm{NH}_{4} \mathrm{OH}\right.$ or TFA) binary mobile phase.

Chick Chorioallantoic Membrane (CAM) Assay. Implantation, Extraction, and Chalkley Score. Animal studies were performed in accordance with the guidelines and regulations laid down in the Animals (Scientific Procedures) Act 1986. CAM model was carried out in accordance with Home Office Approval, UK (Project licensePPL P3E01C456). Chicken eggs were acquired from Medeggs (Norfolk, UK). Eggs were stored in a Hatchmaster incubator (Brinsea, UK) at $37{ }^{\circ} \mathrm{C}$ in a $60 \%$ humidified atmosphere and $1 \mathrm{~h}$ rotation. To ensure the maintenance of a humidified environment in the egg incubator, deionized water (DW) was supplemented every 2 days. Implantation was carried out after 7 days of incubation. To assess embryo viability and development, eggs were candled. A window of $1 \mathrm{~cm}^{2}$ was created with a scalpel onto the egg shell exposing the chorioallantoic membrane. Hydrogels were implanted, and the window was sealed with a sterile Parafilm strip (Bemis, Parafilm M, Laboratory Wrapping Film, Fisher Scientific, UK). Eggs were return to the Hatchmaster incubator for 7 days $\left(37^{\circ} \mathrm{C}\right.$ in a $60 \%$ humidified atmosphere) without rotation. Chalkley scoring was used as previously described ${ }^{3}$ to quantify infiltration of blood vessels through the implanted scaffolds. Implants and blank controls were observed in situ under a stereo light microscope. A total of five independent counts obtained from the number of vessels fitting with the Chalkley graticule projected onto the samples were registered.

Histological Analysis. Integrated hydrogel samples were extracted and fixed in $4 \%$ paraformaldehyde (PFA) overnight. Samples were further embedded in optimum cutting temperature (OCT embedding matrix, CellPath, UK) and stored at $-80^{\circ} \mathrm{C}$. Samples were sectioned using a Cryostat (CM 1850, Leica Biosystems, Germany), and $8 \mu \mathrm{m}$ thick sections were collected using Kawamoto's film method. ${ }^{4}$ Stainings (Goldner's Trichrome and Von Kossa) were subsequently carried out on the cryotape. Sections were mounted using Super Cryomounting Medium (SCMM) type R3 (Section LAB, Co. Ltd. Japan) and UV cured for $30 \mathrm{~min}$ to photopolymerize the SCMM. Slides were imaged the following day using a Zeiss Axiovert 200 (Carl Zeiss, Germany).

\section{ASSOCIATED CONTENT}

\section{(s) Supporting Information}

The Supporting Information is available free of charge at https://pubs.acs.org/doi/10.1021/acsnano.0c09814.

Detailed explanation of the experimental methods and additional figures (PDF)

\section{AUTHOR INFORMATION}

\section{Corresponding Author}

Alvaro Mata - Institute of Bioengineering, Queen Mary University of London, London E1 4NS, U.K.; School of Engineering and Materials Science, Queen Mary University of 
London, London E1 4NS, U.K.; School of Pharmacy, University of Nottingham, Nottingham NG7 2RD, U.K.; Biodiscovery Institute and Department of Chemical and Environmental Engineering, University of Nottingham, Nottingham NG7 2RD, U.K.; Email: a.mata@ nottingham.ac.uk

\section{Authors}

Babatunde O. Okesola - Institute of Bioengineering, Queen Mary University of London, London E1 4NS, U.K.; School of Engineering and Materials Science, Queen Mary University of London, London E1 4NS, U.K.; 이이이.org/0000-00030392-9205

Ana Karen Mendoza-Martinez - Institute of Bioengineering, Queen Mary University of London, London E1 4NS, U.K.; School of Engineering and Materials Science, Queen Mary University of London, London E1 4NS, U.K.

Gianluca Cidonio - Bone and Joint Research Group, Centre for Human Development, Stem Cells and Regeneration, Institute of Developmental Sciences, University of Southampton, Southampton SO16 6YD, U.K.; Center for Life Nano- \& Neuro- Science (CL2NS), Fondazione Istituto Italiano di Tecnologia, 00161 Rome, Italy

Burak Derkus - Institute of Bioengineering, Queen Mary University of London, London E1 4NS, U.K.; School of Engineering and Materials Science, Queen Mary University of London, London E1 4NS, U.K.; Department of Chemistry, Faculty of Science, Ankara University, 06560 Ankara, Turkey; 이이. orcid.org/0000-0001-5558-0995

Delali K. Boccorh - Department of Pure and Applied Chemistry, Technology and Innovation Centre, University of Strathclyde, Glasgow G1 1RD, U.K.

David Osuna de la Peña - School of Engineering and Materials Science, Queen Mary University of London, London E1 4NS, U.K.

Sherif Elsharkawy - Centre for Oral, Clinical, and Translational Sciences, Faculty of Dentistry, Oral, and Craniofacial Sciences, King's College London, London SE1 1UL, U.K.

Yuanhao Wu - School of Pharmacy, University of Nottingham, Nottingham NG7 2RD, U.K.; Biodiscovery Institute, University of Nottingham, Nottingham NG7 2RD, U.K.

Jonathan I. Dawson - Bone and Joint Research Group, Centre for Human Development, Stem Cells and Regeneration, Institute of Developmental Sciences, University of Southampton, Southampton SO16 6YD, U.K.; (1) orcid.org/ 0000-0002-6712-0598

Alastair W. Wark - Department of Pure and Applied Chemistry, Technology and Innovation Centre, University of Strathclyde, Glasgow G1 1RD, U.K.; (1) orcid.org/00000001-8736-7566

Dafna Knani - Department of Biotechnology Engineering, ORT Braude College, Karmiel 2161002, Israel; (1) orcid.org/0000-0002-9490-2819

Dave J. Adams - School of Chemistry, College of Science and Engineering, University of Glasgow, Glasgow G12 8QQ, U.K.; (1) orcid.org/0000-0002-3176-1350

Richard O. C. Oreffo - Bone and Joint Research Group, Centre for Human Development, Stem Cells and Regeneration, Institute of Developmental Sciences, University of Southampton, Southampton SO16 6YD, U.K.; (1) orcid.org/0000-0001-5995-6726
Complete contact information is available at:

https://pubs.acs.org/10.1021/acsnano.0c09814

\section{Notes}

The authors declare no competing financial interest.

\section{ACKNOWLEDGMENTS}

The work was supported by the ERC Starting Grant (STROFUNSCAFF), the Medical Research Council (UK Regenerative Medicine Platform Acellular/Smart Materials-3D Architecture, MR/R015651/1) to A.M., J.I.D., and R.O., and the AO Foundation (AOCMF-17-19M). B.O.O. was supported by the Henry Royce Institute for Advanced Materials, funded through Engineering and Physical Sciences Research Council (EPSRC) grants (EP/R00661X/1, EP/ S019367/1, $\mathrm{EP} / \mathrm{P} 025021 / 1$, and EP/P025498/1). D.J.A. thanks EPSRC for an award of a fellowship (EP/L021978/2). The experiment at the ISIS Neutron and Muon Source was allocated beam time under experiment number 1810221 (DOI: 10.5286/ ISIS.E.90604998) and collected on LARMOR. This work benefited from the SasView software, originally developed by the DANSE project under NSF award DMR-0520547. We thank Vicente Araullo-Peters and Giulia Mastroianni at Nanovision and School of Biological and Chemical Sciences (SBCS), QMUL as well as Janos Kanczler, Bone and Joint Research Group, Southampton for technical support. We thank Sarah Rogers, King Stephen, and Adam Washington from ISIS for SANS experiments. We thank Matthew Smith at Henry Royce Institute, Manchester for HRTEM and EDX analyses. We thank Richard Thorogate at London Centre for Nanotechnology for AFM analyses.

\section{REFERENCES}

(1) Wegst, U. G. K.; Bai, H.; Saiz, E.; Tomsia, A. P.; Ritchie, R. O. Bioinspired Structural Materials. Nat. Mater. 2015, 14, 23-36.

(2) Elsharkawy, S.; Mata, A. Hierarchical Biomineralization: From Nature's Designs to Synthetic Materials for Regenerative Medicine and Dentistry. Adv. Healthcare Mater. 2018, 7, 1800178.

(3) Asenath-Smith, E.; Li, H.; Keene, E. C.; She, Z. W.; Estroff, L. A. Crystal Growth of Calcium Carbonate in Hydrogels as a Model of Biomineralization. Adv. Funct. Mater. 2012, 22, 2891-2914.

(4) Lemloh, M.-L.; Altintoprak, K.; Wege, C.; Weiss, I. M.; Rothenstein, D. Biogenic and Synthetic Peptides with Oppositely Charged Amino Acids as Binding Sites for Mineralization. Materials 2017, 10, 119.

(5) Pohnert, G. Biomineralization in Diatoms Mediated through Peptide- and Polyamine-Assisted Condensation of Silica. Angew. Chem., Int. Ed. 2002, 41, 3167-3169.

(6) Evans, J. S. Composite Materials Design: Biomineralization Proteins and the Guided Assembly and Organization of Biomineral Nanoparticles. Materials 2019, 12, 581.

(7) Pigliacelli, C.; Sánchez-Fernández, R.; García, M. D.; Peinador, C.; Pazos, E. Self-Assembled Peptide-Inorganic Nanoparticle Superstructures: From Component Design to Applications. Chem. Commun. 2020, 56, 8000-8014.

(8) Okesola, B. O.; Suravaram, S. K.; Parkin, A.; Smith, D. K. Selective Extraction and in Situ Reduction of Precious Metal Salts from Model Waste to Generate Hybrid Gels with Embedded Electrocatalytic Nanoparticles. Angew. Chem., Int. Ed. 2016, 55, $183-187$.

(9) Xavier, J. R.; Thakur, T.; Desai, P.; Jaiswal, M. K.; Sears, N.; Cosgriff-Hernandez, E.; Kaunas, R.; Gaharwar, A. K. Bioactive Nanoengineered Hydrogels for Bone Tissue Engineering: A Growth-Factor-Free Approach. ACS Nano 2015, 9, 3109-3118.

(10) Elsharkawy, S.; Al-Jawad, M.; Pantano, M. F.; Tejeda-Montes, E.; Mehta, K.; Jamal, H.; Agarwal, S.; Shuturminska, K.; Rice, A.; 
Tarakina, N. V.; Wilson, R. M.; Bushby, A. J.; Alonso, M.; RodriguezCabello, J. C.; Barbieri, E.; Del Río Hernández, A.; Stevens, M. M.; Pugno, N. M.; Anderson, P.; Mata, A. Protein Disorder-Order Interplay to Guide the Growth of Hierarchical Mineralized Structures. Nat. Commun. 2018, 9, 2145.

(11) Kim, E.; Agarwal, S.; Kim, N.; Hage, F. S.; Leonardo, V.; Gelmi, A.; Stevens, M. M. Bioinspired Fabrication of DNA-Inorganic Hybrid Composites Using Synthetic DNA. ACS Nano 2019, 13, $2888-2900$.

(12) Okesola, B. O.; Ni, S.; Derkus, B.; Galeano, C. C.; Hasan, A.; Wu, Y.; Ramis, J.; Buttery, L.; Dawson, J. I.; D’Este, M.; Oreffo, R. O. C.; Eglin, D.; Sun, H.; Mata, A. Growth-Factor Free Multicomponent Nanocomposite Hydrogels that Stimulate Bone Formation. Adv. Funct. Mater. 2020, 30, 1906205.

(13) Slavik, P.; Smith, D. K. Hybrid Hydrogels Loaded with Palladium Nanoparticles - Catalysts for Environmentally-Friendly Sonogashira and Heck Cross-Coupling Reactions. Tetrahedron 2020, 76,131344 .

(14) Sugawara-Narutaki, A. Bio-Inspired Synthesis of PolymerInorganic Nanocomposite Materials in Mild Aqueous Systems. Polym. J. 2013, 45, 269-276.

(15) Saveleva, M. S.; Eftekhari, K.; Abalymov, A.; Douglas, T. E. L.; Volodkin, D.; Parakhonskiy, B. V.; Skirtach, A. G. Hierarchy of Hybrid Materials - The Place of Inorganics-in-Organics in It, Their Composition and Applications. Front. Chem. 2019, 7, 179.

(16) Kim, Y.-Y.; Ganesan, K.; Yang, P.; Kulak, A. N.; Borukhin, S.; Pechook, S.; Ribeiro, L.; Kröger, R.; Eichhorn, S. J.; Armes, S. P.; Pokroy, B.; Meldrum, F. C. An Artificial Biomineral Formed by Incorporation of Copolymer Micelles in Calcite Crystals. Nat. Mater. 2011, 10, 890-896.

(17) Mann, S. Self-Assembly and Transformation of Hybrid NanoObjects and Nanostructures under Equilibrium and Non-Equilibrium Conditions. Nat. Mater. 2009, 8, 781-792.

(18) Sadasivan, S.; Dujardin, E.; Li, M.; Johnson, C. J.; Mann, S. DNA-Driven Assembly of Mesoporous Silica/Gold Satellite Nanostructures. Small 2005, 1, 103-106.

(19) Nikitin, M. P.; Zdobnova, T. A.; Lukash, S. V.; Stremovskiy, O. A.; Deyev, S. M. Protein-Assisted Self-Assembly of Multifunctional Nanoparticles. Proc. Natl. Acad. Sci. U. S. A. 2010, 107, 5827.

(20) Chan, M. S.; Landig, R.; Choi, J.; Zhou, H.; Liao, X.; Lukin, M. D.; Park, H.; Lo, P. K. Stepwise Ligand-Induced Self-Assembly for Facile Fabrication of Nanodiamond-Gold Nanoparticle Dimers via Non-Covalent Biotin-Streptavidin Interactions. Nano Lett. 2019, 19, 2020-2026.

(21) Xiang, X.-F.; Li, P.-J.; Liu, B.-F. Tuning the Superhydrophobic Properties of Hierarchical Nano-Microstructural Silica Biomorph Arrays Grown at Triphasic Interfaces. Sci. Rep. 2020, 10, 4596.

(22) Campbell, C. J.; Klajn, R.; Fialkowski, M.; Grzybowski, B. A. One-Step Multilevel Microfabrication by Reaction-Diffusion. Langmuir 2005, 21, 418-423.

(23) Smoukov, S. K.; Bishop, K. J. M.; Klajn, R.; Campbell, C. J.; Grzybowski, B. A. Cutting into Solids with Micropatterned Gels. Adv. Mater. 2005, 17, 1361-1365.

(24) Smoukov, S. K.; Grzybowski, B. A. Maskless Microetching of Transparent Conductive Oxides (ITO and $\mathrm{ZnO}$ ) and Semiconductors (GaAs) Based on Reaction-Diffusion. Chem. Mater. 2006, 18, 47224723.

(25) Lovrak, M.; Hendriksen, W. E. J.; Maity, C.; Mytnyk, S.; van Steijn, V.; Eelkema, R.; van Esch, J. H. Free-Standing Supramolecular Hydrogel Objects by Reaction-Diffusion. Nat. Commun. 2017, 8, 15317.

(26) Kurylo, I.; Gines, G.; Rondelez, Y.; Coffinier, Y.; Vlandas, A. Spatiotemporal Control of DNA-Based Chemical Reaction Network via Electrochemical Activation in Microfluidics. Sci. Rep. 2018, 8, 6396.

(27) Luo, H.; Leprince-Wang, Y.; Jing, G. Tunable Growth of $\mathrm{ZnO}$ Nanostructures on the Inner Wall of Capillary Tubes. J. Phys. Chem. C 2019, 123, 7408-7415.
(28) Campbell, C. J.; Smoukov, S. K.; Bishop, K. J. M.; Baker, E.; Grzybowski, B. A. Direct Printing of 3D and Curvilinear MicrometerSized Architectures into Solid Substrates with Sub-Micrometer Resolution. Adv. Mater. 2006, 18, 2004-2008.

(29) Kleiman, M.; Brubaker, K. S.; Nguyen, D. T.; Esser-Kahn, A. P. Bio-Inspired Morphogenesis Using Microvascular Networks and Reaction-Diffusion. Chem. Mater. 2015, 27, 4871-4876.

(30) Nakouzi, E.; Steinbock, O. Self-Organization in Precipitation Reactions Far from the Equilibrium. Sci. Adv. 2016, 2, No. e1601144.

(31) Okesola, B. O.; Mata, A. Multicomponent Self-Assembly as a Tool to Harness New Properties from Peptides and Proteins in Material Design. Chem. Soc. Rev. 2018, 47, 3721-3736.

(32) Gaharwar, A. K.; Cross, L. M.; Peak, C. W.; Gold, K.; Carrow, J. K.; Brokesh, A.; Singh, K. A. 2D Nanoclay for Biomedical Applications: Regenerative Medicine, Therapeutic Delivery, and Additive Manufacturing. Adv. Mater. 2019, 31, 1900332.

(33) Mousa, M.; Evan, N. D.; Oreffo, R. O. C.; Dawson, J. I. Clay Nanoparticles for Regenerative Medicine and Biomaterial Design: A Review of Clay Bioactivity. Biomaterials 2018, 159, 204-214.

(34) Gaharwar, A. K.; Mukundan, S.; Karaca, E.; Dolatshahi-Pirouz, A.; Patel, A.; Rangarajan, K.; Mihaila, S. M.; Iviglia, G.; Zhang, H.; Khademhosseini, A. Nanoclay-Enriched $\operatorname{Poly}(\varepsilon$-caprolactone $)$ Electrospun Scaffolds for Osteogenic Differentiation of Human Mesenchymal Stem Cells. Tissue Eng., Part A 2014, 20, 2088-2101.

(35) Kerativitayanan, P.; Gaharwar, A. K. Elastomeric and Mechanically Stiff Nanocomposites from Poly(Glycerol Sebacate) and Bioactive Nanosilicates. Acta Biomater. 2015, 26, 34-44.

(36) Nojoomi, A.; Tamjid, E.; Simchi, A.; Bonakdar, S.; Stroeve, P. Injectable Polyethylene Glycol-Laponite Composite Hydrogels as Articular Cartilage Scaffolds with Superior Mechanical and Rheological Properties. Int. J. Polym. Mater. 2017, 66, 105-114.

(37) Basu, S.; Pacelli, S.; Feng, Y.; Lu, Q.; Wang, J.; Paul, A. Harnessing the Non-Covalent Interactions of DNA Backbone with 2D Silicate Nanodisks to Fabricate Injectable Therapeutic Hydrogels. ACS Nano 2018, 12, 9866-9880.

(38) Su, D.; Jiang, L.; Chen, X.; Dong, J.; Shao, Z. Enhancing the Gelation and Bioactivity of Injectable Silk Fibroin Hydrogel with Laponite Nanoplatelets. ACS Appl. Mater. Interfaces 2016, 8, 96199628.

(39) Liu, B.; Li, J.; Lei, X.; Miao, S.; Zhang, S.; Cheng, P.; Song, Y.; Wu, H.; Gao, Y.; Bi, L.; Pei, G. Cell-Loaded Injectable Gelatin/ Alginate/Laponite ${ }^{\circledR}$ Nanocomposite Hydrogel Promotes Bone Healing in a Critical-Size Rat Calvarial Defect Model. RSC Adv. 2020, 10, 25652-25661.

(40) Koshy, S. T.; Zhang, D. K.Y.; Grolman, J. M.; Stafford, A. G.; Mooney, D. J. Injectable Nanocomposite Cryogels for Versatile Protein Drug Delivery. Acta Biomater. 2018, 65, 36-43.

(41) Page, D. J.; Clarkin, C. E.; Mani, R.; Khan, N. A.; Dawson, J. I.; Evans, N. D. Injectable Nanoclay Gels for Angiogenesis. Acta Biomater. 2019, 100, 378-387.

(42) Capito, R. M.; Azevedo, H. S.; Velichko, Y. S.; Mata, A.; Stupp, S. I. Self-Assembly of Large and Small Molecules into Hierarchically Ordered Sacs and Membranes. Science 2008, 319, 1812.

(43) Inostroza-Brito, K. E.; Collin, E.; Siton-Mendelson, O.; Smith, K. H.; Monge-Marcet, A.; Ferreira, D. S.; Rodríguez, R. P.; Alonso, M.; Rodríguez-Cabello, J. C.; Reis, R. L.; Sagués, F.; Botto, L.; Bitton, R.; Azevedo, H. S.; Mata, A. Co-Assembly, Spatiotemporal Control and Morphogenesis of a Hybrid Protein-Peptide System. Nat. Chem. 2015, 7, 897-904.

(44) Hedegaard, C. L.; Collin, E. C.; Redondo-Gómez, C.; Nguyen, L. T. H.; Ng, K. W.; Castrejón-Pita, A. A.; Castrejón-Pita, J. R.; Mata, A. Hydrodynamically Guided Hierarchical Self-Assembly of PeptideProtein Bioinks. Adv. Funct. Mater. 2018, 28, 1703716.

(45) Okesola, B. O.; Lau, H. K.; Derkus, B.; Boccorh, D. K.; Wu, Y.; Wark, A. W.; Kiick, K. L.; Mata, A. Covalent Co-Assembly between Resilin-Like Polypeptide and Peptide Amphiphile into Hydrogels with Controlled Nanostructure and Improved Mechanical Properties. Biomater. Sci. 2020, 8, 846-857. 
(46) Okesola, B. O.; Wu, Y.; Derkus, B.; Gani, S.; Wu, D.; Knani, D.; Smith, D. K.; Adams, D. J.; Mata, A. Supramolecular Self-Assembly to Control Structural and Biological Properties of Multicomponent Hydrogels. Chem. Mater. 2019, 31, 7883-7897.

(47) Zechel, S.; Hager, D. M.; Priemel, T.; Harrington, J. M. Healing through Histidine: Bioinspired Pathways to Self-healing Polymers via Imidazole-Metal Coordination. Biomimetics 2019, 4, 20.

(48) Wang, Q.; Mynar, J. L.; Yoshida, M.; Lee, E.; Lee, M.; Okuro, K.; Kinbara, K.; Aida, T. High-Water-Content Mouldable Hydrogels by Mixing Clay and a Dendritic Molecular Binder. Nature 2010, 463, 339-343.

(49) Mata, A.; Palmer, L.; Tejeda-Montes, E.; Stupp, S. I. Design of Biomolecules for Nanoengineered Biomaterials for Regenerative Medicine. In Nanotechnology in Regenerative Medicine: Methods and Protocols; Navarro, M., Planell, J. A., Eds.; Humana Press: Totowa, 2012; pp 39-49.

(50) Shi, J.; Wang, C.; Ngai, T.; Lin, W. Diffusion and Binding of Laponite Clay Nanoparticles into Collagen Fibers for the Formation of Leather Matrix. Langmuir 2018, 34, 7379-7385.

(51) BIOVIA Materials Studio - An Integrated, Multi-Scale Modeling Environment. Accessed 2020-01-25. www.3ds.com/ products-services/biovia/products/molecular-modeling-simulation/ biovia-materials-studio/.

(52) Appel, E. A.; Tibbitt, M. W.; Webber, M. J.; Mattix, B. A.; Veiseh, O.; Langer, R. Self-Assembled Hydrogels Utilizing PolymerNanoparticle Interactions. Nat. Commun. 2015, 6, 6295.

(53) Ligorio, C.; Zhou, M.; Wychowaniec, J. K.; Zhu, X.; Bartlam, C.; Miller, A. F.; Vijayaraghavan, A.; Hoyland, J. A.; Saiani, A. Graphene Oxide Containing Self-Assembling Peptide Hybrid Hydrogels as a Potential 3D Injectable Cell Delivery Platform for Intervertebral Disc Repair Applications. Acta Biomater. 2019, 92, 92-103.

(54) Brown, N.; Lei, J.; Zhan, C.; Shimon, L. J. W.; AdlerAbramovich, L.; Wei, G.; Gazit, E. Structural Polymorphism in a SelfAssembled Tri-Aromatic Peptide System. ACS Nano 2018, 12, 32533262.

(55) Ren, Y.; Ma, Z.; Morris, R. E.; Liu, Z.; Jiao, F.; Dai, S.; Bruce, P. G. A Solid with a Hierarchical Tetramodal Micro-Meso-Macro Pore Size Distribution. Nat. Commun. 2013, 4, 2015.

(56) Ng, K. C.; Burhan, M.; Shahzad, M. W.; Ismail, A. B. A Universal Isotherm Model to Capture Adsorption Uptake and Energy Distribution of Porous Heterogeneous Surface. Sci. Rep. 2017, 7, 10634.

(57) Cao, Y.; Mei, M. L.; Li, Q.-L.; Lo, E. C. M.; Chu, C. H. Polydopamine-Induced Tooth Remineralization. ACS Appl. Mater. Interfaces 2014, 6, 410-420.

(58) Habraken, W. J. E. M.; Tao, J.; Brylka, L. J.; Friedrich, H.; Bertinetti, L.; Schenk, A. S.; Verch, A.; Dmitrovic, V.; Bomans, P. H. H.; Frederik, P. M.; Laven, J.; van der Schoot, P.; Aichmayer, B.; de With, G.; DeYoreo, J. J.; Sommerdijk, N. A. J. M. Ion-Association Complexes Unite Classical and Non-Classical Theories for the Biomimetic Nucleation of Calcium Phosphate. Nat. Commun. 2013, 4, 1507.

(59) Mann, S. The Chemistry of Form. Angew. Chem., Int. Ed. 2000, 39, 3392-3406.

(60) De Yoreo, J. J.; Gilbert, P. U. P. A.; Sommerdijk, N. A. J. M.; Penn, R. L.; Whitelam, S.; Joester, D.; Zhang, H.; Rimer, J. D.; Navrotsky, A.; Banfield, J. F.; Wallace, A. F.; Michel, F. M.; Meldrum, F. C.; Cölfen, H.; Dove, P. M. Crystallization by Particle Attachment in Synthetic, Biogenic, and Geologic Environments. Science 2015, 349, aaa6760.

(61) Kumar, M.; Luo, H.; Román-Leshkov, Y.; Rimer, J. D. SSZ-13 Crystallization by Particle Attachment and Deterministic Pathways to Crystal Size Control. J. Am. Chem. Soc. 2015, 137, 13007-13017.

(62) Asenath-Smith, E.; Hovden, R.; Kourkoutis, L. F.; Estroff, L. A. Hierarchically Structured Hematite Architectures Achieved by Growth in a Silica Hydrogel. J. Am. Chem. Soc. 2015, 137, 51845192.
(63) Seto, J.; Ma, Y.; Davis, S. A.; Meldrum, F.; Gourrier, A.; Kim, Y.-Y.; Schilde, U.; Sztucki, M.; Burghammer, M.; Maltsev, S.; Jäger, C.; Cölfen, H. Structure-Property Relationships of a Biological Mesocrystal in the Adult Sea Urchin Spine. Proc. Natl. Acad. Sci. U. S. A. 2012, 109, 3699.

(64) Sargeant, T. D.; Aparicio, C.; Goldberger, J. E.; Cui, H.; Stupp, S. I. Mineralization of Peptide Amphiphiles Nanofibers and Its Effect on Differentiation of Human Mesenchymal Stem Cells. Acta Biomater. 2012, 8, 2456-2465.

(65) Liu, Y.; Meng, H.; Konst, S.; Sarmiento, R.; Rajachar, R.; Lee, B. P. Injectable Dopamine-Modified Polyethylene Glycol Nanocomposite Hydrogel with Enhanced Adhesive Property and Bioactivity. ACS Appl. Mater. Interfaces 2014, 6, 16982-16992.

(66) de Aza, P. N.; Santos, C.; Pazo, A.; de Aza, S.; Cuscó, R.; Artús, L. Vibrational Properties of Calcium Phosphate Compounds. 1. Raman Spectrum of $\beta$-Tricalcium Phosphate. Chem. Mater. 1997, 9, 912-915.

(67) Kurouski, D.; Van Duynea, R. P.; Lednev, I. K. Exploring the Structure and Formation Mechanism of Amyloid Fibrils by Raman Spectroscopy: A Review. Analyst 2015, 140, 4967-4980.

(68) Nakayama, M.; Kajiyama, S.; Kumamoto, A.; Nishimura, T.; Ikuhara, Y.; Yamato, M.; Kato, T. Stimuli-Responsive Hydroxyapatite Liquid Crystal with Macroscopically Controllable Ordering and Magneto-Optical Functions. Nat. Commun. 2018, 9, 568.

(69) Marshall, K. M.; Kanczler, J. M.; Oreffo, R. O. C. J. Evolving Applications of the Egg: Chorioallantoic Membrane Assay and ex Vivo Organotypic Culture of Materials for Bone Tissue Engineering. $J$. Tissue Eng. 2020, 11, 1-25. 